\title{
Solution-diffusion properties of C4H6 and N2 molecules in zeolitic-imidazolate framework based mixed matrix membranes
}

\author{
Ihtisham Haq ${ }^{1}$, Tao Wang ${ }^{2}$, Ao-shuai Zhang ${ }^{2}$, Rehan Khan ${ }^{2}$, Li-Hao Xu², and Zhi-Ping \\ Zhao $^{2}$ \\ ${ }^{1}$ Beijing Institute of Technology \\ ${ }^{2}$ Beijing institute of technology
}

May 21, 2020

\begin{abstract}
This research investigated comprehensively the fundamental science of estimating sorption, diffusion, and permeation of 1,3butadiene and nitrogen in polydimethylsiloxane (PDMS), and zeolitic imidazolate frameworks (ZIFs) filled mixed-matrix membranes (MMMs). ZIF-8 and Ni-ZIF-8 nanocrystals were synthesized at room temperature and incorporated into PDMS at different loadings. The physical and chemical properties of both ZIFs have been studied by XRD, FTIR, SEM, EDX, and TGA, respectively. Synthesized Ni-ZIF-8/PDMS MMMs demonstrated homogeneous particle dispersion, improved particle-polymer adhesion, and better butadiene permeation under various testing conditions. 15\% Ni-ZIF-8/PDMS MMM exhibits 1,3-butadiene permeance 323 GPU, which improved by $60 \%$ compared with PDMS, and 32\% with 15\% ZIF-8/PDMS MMM, while BD/N2 ideal selectivity of 19.5 was higher $81 \%$ than PDMS, and $32 \%$ than $15 \%$ ZIF-8/PDMS MMM at $40^{\circ} \mathrm{C}$ and 1.5 bar. Additionally, Ni-ZIF-8/PDMS MMMs have improved the solution-diffusion properties of butadiene and decreased sorption enthalpy, entropy change, activation energies of diffusion, and permeability from PDMS and ZIF-8/PDMS.
\end{abstract}

\section{Keywords,}

Zeolitic imidazolate frameworks (ZIFs), Mixed-matrix membranes, 1,3-Butadiene, Solutiondiffusion properties, Activation energies

\section{Introduction}

1,3-Butadiene (BD) is a very versatile organic raw material, a petrochemical-based VOC, used as a monomer for the manufacture of synthetic rubber ${ }^{1}$. Due to the presence of conjugated double bonds in its molecule, addition, cyclization, substitution, and polymerization reactions can take place, which makes this VOC a widely used compound in synthetic rubber and organic synthesis ${ }^{2}$. Due to its high consumption for the synthetic rubber industries, it became so essential to control it from the tail gases of synthetic rubber plants. In addition, the international agency for research on cancer has designated 1,3-Butadiene as a possible carcinogenic material, while environmental protection agency (EPA) has listed it as the $10^{\text {th }}$ most carcinogenic material ${ }^{3}$. Butadiene mainly mixed with the nitrogen during its post-treatment, i.e., drying process, where butadiene evaporates from the synthesized rubber and mixed with the tail gas of the plant. At present, the catalytic oxidation method used to remove the $\mathrm{BD}$ from tail gas before emitted to air, which completely converted the VOCs into $\mathrm{CO}_{2}$ and $\mathrm{H}_{2} \mathrm{O}$. Although catalytic oxidation removed $\mathrm{BD}$ from tail gas of the synthetic rubber plant, it is a destructive method to control the VOC emission so that there must be some constructive method required, which can effectively recover the BD from tail gas and can be reused as well.

Membrane-based technology is one of the best possible solutions for complete VOC recovery. The membrane separation process developed over the last decades and considered to be very attractive due to its ease of 
operation, small footprint, low energy usage, easily scale up production, and more significantly, it offers the ability to meet future stricter environmental limits ${ }^{4}$. In the recent works, MMMs showed good results for many applications such as water separation, flue gas purification, petrochemical separation, and natural gas separation ${ }^{5-9}$. The synthesis of mixed matrix membranes (MMMs) is the most promising approach for using both organic and inorganic material properties at the same time, which also improves gas transport properties and may surpass the Robeson upper bound limit ${ }^{4,10}$. It involves the fabrication of composite membranes between polymeric materials as a base, and inorganic material as filler particles. Theoretically, by use of MMMs, benefits of both polymer and inorganic content can be used at the same time. Moore et al. identified the different non-ideal structures in MMMs such as rigidified polymer layer around the inorganic fillers, interface voids or sieve in-a-cage, and particle pore blockage ${ }^{11}$. In order to surpass the Robeson upper bound, the MMMs structure must be defect-free at the polymer/filler interface. For overcoming the poor adhesion, it requires careful selection of the filler and polymer, which must have good interaction with each other. However, the selection of fillers and polymers is limited to form a defect-free interface on the basis of these factors ${ }^{12}$. Polydimethylsiloxane $(\mathrm{PDMS})^{13-15}$, polyether block amide (PEBA) ${ }^{5,16}$, matrimid ${ }^{17}$, polymers of intrinsic micro porosity $(\mathrm{PIM})^{18}$, polyethylene oxide $(\mathrm{PEO})^{19}$, polyvinyl alcohol $(\mathrm{PVA})^{20}$, and several polyimides ${ }^{21,22}$ are polymers that have been commonly used in the fabrication of MMMs, while carbon molecular sieves ${ }^{23}$, zeolites ${ }^{24}$, carbon nanotubes ${ }^{13}$, graphene oxide $(\mathrm{GO})^{25,26}$, and metal-organic frameworks $(\mathrm{MOFs})^{27,28}$, are the most extensively used nano porous materials as fillers in MMMs.

There is no data available for the separation of BD from its nitrogen mixture through the membrane separation process in the literature. Baker et al. stated that rubbery membranes are beneficial for the $99 \%$ recovery of hydrocarbons with a suitable process design ${ }^{29}$. Polydimethylsiloxane (PDMS) is a rubbery polymer and well-known membrane material due to its excellent chain flexibility makes it very permeable even at high operating temperature and low trans-membrane pressure. By the end of the last century, PDMS predominated and widely studied polymer for hydrocarbon separation. Past results have shown that the PDMS membrane has excellent potential for recovering hydrocarbons from nitrogen mixed streams ${ }^{30-32}$. Additionally, it is worth noticing that the 1,3-butadiene permeation through rubbery polymers such as PDMS is superior to its permeation through any glassy polymer since there exist two to three orders of magnitude difference in permeation ${ }^{33}$.

Zeolite imidazolate framework (ZIF) is a subfamily of MOFs, which is coordinated between the transition metals such as zinc and cobalt and imidazole linkers ${ }^{34}$. In recent years, ZIFs have engrossed deep interest as a versatile crystalline porous material for gas storage and gas separation applications. The highly crystalline and nano-porous structures of ZIFs, make them an ideal candidate for the separation of small kinetic diameters gas particles. The imidazolate linkers in ZIFs frameworks made them more hydrophobic as compared with any other filler types, which deliberates the excellent interfacial compatibility between polymers and $\mathrm{ZIFs}^{35}$. Moreover, the imidazolate linker property of structural flexibility makes its role more essential and vital as it involves in gate opening effect of ZIFs for large molecules than the pores of ZIFs ${ }^{36}$. ZIF-8 is the most studied ZIF material among all of its types in MMMs for the gas separation ${ }^{34,37,38}$. It is worth noticing that; besides the extensively researched linker-substituted effect, metal-substitution also influences the molecular sieving efficiency of the associated ZIF crystals. By introducing a second metal ion in the MOF cluster, the stability and affinity towards the target gases can be largely improved ${ }^{39-41}$. Many recent works have reported in which researchers have used mixed metal MOFs by adding another metal in the ZIF-8 crystal for properties enhancement ${ }^{41}$. The results were improved by the addition of another metal in the ZIF- 8 cluster. To the best of our knowledge, the separation of low boiling point hydrocarbons (gases at room temperature) from permanent gases, using ZIF-based membranes, is very limited. Just one study reported by Fang et al. in which they used ZIF-8/PDMS/PVDF MMMs to separate propane from nitrogen mixture ${ }^{42}$. The $\mathrm{C}_{3} \mathrm{H}_{8} / \mathrm{N}_{2}$ selectivity was $38 \%$ improved by using $10 \%$ ZIF-8-MMMs relative to the pure PDMS membrane. Therefore, the ZIF- 8 based MMMs should be further examined for the hydrocarbon separation from permanent gases.

This work, for the first time, employed Zn/Ni-ZIF-8 as a filler for the preparation of MMMs with PDMS polymer to improve the gas-separation properties of the membranes for the separation of $\mathrm{BD} / \mathrm{N}_{2}$. Ni-ZIF-8 has the same zeolite nets-sodalite (SOD) topological structure with ZIF-8, while Ni just partially substitutes 
the $\mathrm{ZnN}_{4}$ in the backbone of the ZIF structure, and tetrahedrally connected with the nitrogen, which makes it a very stable structure of four coordinated with Ni centers. Mixed metal ZIF was used to enhance BD affinity, which resulted in a sufficient adsorption capacity due to the presence of two metals in its cluster. The difference of gas permeation results was tested for single and mixed metal ZIF-8 in their MMMs. The effects of Ni-ZIF-8 loading on the microstructure of the membranes were investigated. The influence of Ni-ZIF-8 nanoparticle loadings feed temperature, and feed pressure on gas-permeation performance were also observed. Importantly, the gas transport properties across these MMMs for 1,3-butadiene and $\mathrm{N}_{2}$ were investigated on a preliminary basis by exploring the effects of different MMMs. This study will introduce the permeation and solution-diffusion properties of Ni-ZIF-8/PDMS MMMs for the removal of BD from nitrogen mixture, which will also provide a scientific contribution in the literature.

\section{Materials}

Zinc nitrate hexahydrate $\left(\mathrm{Zn}\left(\mathrm{NO}_{3}\right)_{2} \cdot 6 \mathrm{H}_{2} \mathrm{O}, 99.99 \%\right)$, 2-methylimidazole (Hmim, 98\%), and Tetraethyl orthosilicate (TEOS, 98\%) were supplied by Shanghai Aladdin Industrial Corporation China. PVDF porous support membrane was prepared using the nonsolvent-induced phase separation (NIPS) method. Dibutyltin dilaurate (DBTDL, [?]96\%) was supplied by Tianjin Guangfu Fine Chemical Research Institute China. Hydroxyl-terminated PDMS (viscosity, $50000 \mathrm{mPa}$ ) was purchased from Beijing Chemical Works China. Methanol of analytical grade was purchased from Beijing Tongguang Fine Chemical Company China. Butadiene and nitrogen pure gases (99.999\%) were supplied by Shanxi Hongwei Gas Company China. All reagents were used as received without any further purification.

\section{Synthesis of ZIF-8}

ZIF-8 nanoparticles were synthesized by following the method, which is available in the literature ${ }^{43}$. Typically, $1.8 \mathrm{~g}$ of $\mathrm{Zn}\left(\mathrm{NO}_{3}\right)_{2} * 6 \mathrm{H}_{2} \mathrm{O}$ and $4.0 \mathrm{~g}$ of 2-methylimidazole were mixed in $100 \mathrm{ml}$ methanol separately, then they were mixed and remained stirred for the next $1 \mathrm{hr}$. Afterward, the white product was centrifuged (TG16-WS CENCE Company Ltd. P.R.China) and rinsed with methanol two times and dried in an oven at 60degC.

\section{Synthesis of Ni-ZIF-8}

Ni-ZIF-8 nanoparticles were made according to the method available elsewhere ${ }^{44}$. $\mathrm{Zn}\left(\mathrm{NO}_{3}\right)_{2} \cdot 6 \mathrm{H}_{2} \mathrm{O}(1.188$ g) and $\mathrm{Ni}\left(\mathrm{NO}_{3}\right)_{2} \cdot 6 \mathrm{H}_{2} \mathrm{O}(1.172 \mathrm{~g})$ were mixed in $200 \mathrm{ml}$ methanol separately and mixed with the solution of 2-methylimidazole $(5.296 \mathrm{~g})$ in methanol $(100 \mathrm{ml})$, which was stirred for next $24 \mathrm{hrs}$ at ambient conditions. Then violet color powder was washed three times with methanol and dried in a vacuum oven at 80degC.

\section{MMMs preparation}

The membrane was prepared by the solution casting method. First of all, ZIFs nanoparticles were added in n-heptane and stirred it for 30 minutes and then sonicated for the next 30 minutes. The same steps were repeated three times simultaneously. Then the 10\% PDMS of its total weight was added for "priming" in the ZIFs/n-heptane solution (wPDMS:wn-heptane $=0.1: 9$ ) and let it further stirred for next $3 \mathrm{hrs}$. Subsequently, more PDMS was added after each $3 \mathrm{~h}$ with different amounts of PDMS (20, 30 and 40\%) and then remained it stirred for next $12 \mathrm{~h}$. TEOS (crosslinker) was added with definite ratio (wPDMS:wnheptane:wTEOS:wdibutyltin dilaurate $=1: 9: 0.1: 0.01)$. After $2 \mathrm{~h}$, the catalyst (DD) was added and stirred the solution for the next 20 minutes. Moreover, the casting solution was placed in the sonicator for 20 minutes before casting the membrane, as this method is helpful for the good dispersion of fillers within the polymer matrix. Then the slightly viscous solution was cast over PVDF support following the solution casting method. The membranes were dried in the open air for $3 \mathrm{~h}$ and kept in an oven at 120degC for the next $4 \mathrm{~h}$ for the sake of crosslinking and the complete removal of solvents from it. By following the same method, we have synthesized the pure PDMS/PVDF membrane without any loading of ZIFs. The ZIFs loading was defined by using the following equation,

$w_{\mathrm{ZIF}}=\frac{m_{\mathrm{ZIF}}}{m_{\mathrm{PDMS}}} \times 100(1)$ 
here, $w$ ZIF represents the loading of ZIFs nanoparticles in the MMMs, $m$ ZIF, and $m$ PDMS represents the mass of ZIFs and mass of PDMS added in the casting solution, respectively.

The Ni-ZIF-8 loading in these MMMs were $0,5,10,15$ and $20 \%$ respectively and resultant membranes were labeled as, X-Ni-ZIF-8 MMM $(\mathrm{X}=0,5,10,15,20)$. For the sake of comparison with ZIF-8 based MMMs, we made the same loading MMMs of ZIF- 8 by following the same method.

\section{Gas permeation test}

The gas permeation tests were conducted at different temperatures and pressures on self-designed equipment, which was based on constant pressure and variable volume (Figure. S1), and gas permeation testing details are given in supplementary information. In this work, we measured gas transportation across the membrane in "permeance" as it is independent of the membrane thickness and membrane module. Simple flat sheet membranes were used in this work for the separation of butadiene and nitrogen, while different membrane modules widely used in industries for getting better results than the flat sheet membranes. Gas permeance of both the gases was measured, and all the readings were repeated at least five times and reported an average value of it. The gas permeance $(J)$ was calculated by using the following equation,

$J=\frac{q}{p \times A}(2)$

where $q$ represents the flow rate $\left(\mathrm{cm}^{3} / \mathrm{sec}\right)$, which was measured by a bubble flow meter, $p$ used for the trans-membrane pressure $(\mathrm{cm} \mathrm{Hg})$, and $A$ is used for active membrane surface area $\left(\mathrm{cm}^{2}\right)$. The unit used for permeance was GPU (gas permeation unit) $\left(1 \times 10^{-6} \mathrm{~cm}^{3} \mathrm{~cm}^{-2} \mathrm{sec}^{-1} \mathrm{cmHg}^{-1}\right)$.

The permeance is related to the permeability by using the following equation,

$$
P=J \times l
$$

here, $P$ is used for permeability $\left(1 \times 10^{-10} \mathrm{~cm}^{3} \mathrm{~cm} \mathrm{~cm}^{-2} \mathrm{sec}^{-1} \mathrm{cmHg}^{-1}\right)$, and $l$ is for membrane active thickness $(\mathrm{cm})$.

The ideal permeation selectivity $\left(a_{\mathrm{P}}\right)$ was measured by the permeation ratios of pure gases,

$\alpha_{P}=\frac{P_{\mathrm{BD}}}{P_{N 2}}(4)$

here $P$ BD and $P$ N2 showing the permeabilities of butadiene and nitrogen, respectively.

\section{Gas sorption test}

The sorption analysis of pure gases was conducted by using an isothermal equipment "intelligent gravimetric analysis" (IGA) (IGA100C Hiden Isochema Ltd. England) at different temperatures. The gas sorption values were taken volumetrically for pure PDMS and MMMs. Before testing the sorption analysis, the pure PDMS and Ni-ZIF-8 MMMs without PVDF support were placed in the oven at $120^{\circ} \mathrm{C}$ for $6 \mathrm{~h}$ for the complete removal of any adsorbed moisture or solvent from it. The detailed method of the sorption tests is given in supplementary information.

The solubility is the penetrant property to dissolve with the membrane. The solution coefficient expresses the solubility of the component in the medium, which is a dissolution property of components. . The solution coefficient (S) of gas, can be described as the gas concentration divided by pressure of the gas at the equilibrium. So,

$S=\frac{C}{P}(5)$

here $S, \mathrm{C}$, and $P$ representing the solution coefficient $\left(\mathrm{cm}^{3}(\mathrm{STP}) \mathrm{cm}^{-3}\right.$ membrane bar-1), gas concentration $\left(\mathrm{cm}^{3}\right.$ (STP) $\mathrm{cm}^{-3}$ membrane), and pressure at equilibrium (bar), respectively. The solution selectivity $(a \mathrm{~s})$ was measured by using the following equation,

$\alpha_{S}=\frac{S_{\mathrm{BD}}}{S_{N 2}}(6)$ 
here $S_{\mathrm{BD}}$ and $S_{\mathrm{N} 2}$ representing the solution coefficients of butadiene and nitrogen, respectively.

Diffusivity is an important parameter indicative of the penetrant mobility across the membrane based on its concentration difference. The diffusion coefficient expresses the speed of diffusion of components in medium, which is a transfer attribute of components. Through the solution coefficient and permeability values, diffusion coefficient $(D)$ was measured by using the equation,

$D=\frac{P}{S}(7)$

here $D$ representing the diffusion coefficient $\left(\mathrm{cm}^{2} \mathrm{~s}^{-1}\right)$ of the gas, $P$, and $S$ showing the permeability (Barrer) of the gas, and solution coefficient $\left(\mathrm{cm}^{3}\right.$ (STP) $\mathrm{cm}^{-3}$ membrane $\left.\mathrm{cmHg}^{-1}\right)$, respectively.

The diffusion selectivity $\left(a_{\mathrm{D}}\right)$ was measured by using the following equation,

$\alpha_{D}=\frac{D_{\mathrm{BD}}}{D_{N 2}}(8)$

here $D$ BD and $D$ N2 showing the diffusion coefficients of butadiene and nitrogen, respectively.

\section{ZIF nanoparticles and membrane characterizations}

Powder X-ray diffraction (PXRD) and Wide-angle X-ray diffraction (WXRD) patterns were determined for ZIFs nanoparticles and membranes by using an X-ray diffractometer (Ultima IV, Japan) with $\mathrm{Cu}-\mathrm{K} \alpha \mathrm{X}$ ray radiation $(\lambda=1.54056 \AA)$ from 5 to $40^{\circ}$ at the rate of $5^{\circ} \mathrm{min}^{-1}$, respectively. For testing the textural properties of synthesized ZIFs materials surface area analyzer (BELSORP Japan) was used by $\mathrm{N}_{2}$ adsorption and desorption at $77 \mathrm{~K}$. The surface areas of ZIF-8 and Ni-ZIF-8 were calculated by the use of standard Brunauer-Emmett-Teller (BET) equation. Before measuring the surface area, the samples were pre-dried at $150^{\circ} \mathrm{C}$ for 6 hrs under a continuous flow of $\mathrm{N}_{2}$. Morphologies and Energy disperse X-ray spectroscopy (EDXS) of the ZIFs particles, and MMMs were observed through a field-emission scanning electron microscope (FESEM, JSM-7500F, JEOL, Japan), which was operated at $5 \mathrm{kV}$. FTIR spectrum was plotted by using ATRFTIR (Thermo Nicolet IS10, USA) by setting the range of $4000-500 \mathrm{~cm}^{-1}$ with a resolution of $4 \mathrm{~cm}^{-1}$. Thermal stabilities analysis of the nanoparticles and MMMs were analyzed by thermogravimetric analysis (TGA-60 Shimadzu Japan) in the range of 30 to $800^{\circ} \mathrm{C}$ with a ramp rate of $5^{\circ} \mathrm{C} \mathrm{min}^{-1}$, under the continuous flow of nitrogen. The same instrument (TGA-60 Shimadzu Japan) was used for differential scanning calorimetry (DSC) analysis of MMMs.

\section{Results and discussion}

\section{Characterization of ZIFs nanoparticles and MMMs}

In order to obtain the crystal structure of both ZIFs nanoparticles, powder X-ray diffraction (PXRD) analysis was performed. Figure 1(a) shows the PXRD pattern of the simulated ZIF-8, synthesized ZIF-8, and synthesized Ni-ZIF-8, respectively. It can be seen that the main peaks of the synthesized ZIF-8 and Ni-ZIF-8 are very clear and identical with simulated ZIF-8, which proves its structure and phase purity ${ }^{44}$. Figure 1 (b) shows the $\mathrm{N}_{2}$ adsorption-desorption isotherms at $77 \mathrm{~K}$ for Ni-ZIF-8 and ZIF-8. The overlapping of adsorptiondesorption isotherm confirmed its microporous structure as well. BET analysis provided the specific surface areas of ZIF-8 $\left(1420 \mathrm{~m}^{2} / \mathrm{g}\right)$, and Ni-ZIF-8 $\left(1700 \mathrm{~m}^{2} / \mathrm{g}\right)$, while the micropore volumes were $0.58 \mathrm{~cm}^{3}$, and $0.66 \mathrm{~cm}^{3}$ of ZIF-8 and Ni-ZIF-8, respectively. The specific surface areas of ZIFs depend upon different synthesis methods, such as solvent used for synthesis, organic linker, and the synthesis process (solvothermal or hydrothermal). The BET surface area, pore size, and pore volume of both ZIFs are well-matched with the reported work ${ }^{37}$. The SEM images of both ZIFs are given in Figure S2, which confirms that both ZIFs have spherical shapes and homogeneous particle sizes. Ni-ZIF-8 has an average particle size of $40 \mathrm{~nm}$, while ZIF-8 has the $60 \mathrm{~nm}$ (Figure S4). The EDX results (Figure S3) revealed the exact percentage of $\mathrm{Zn}, \mathrm{Ni}, \mathrm{N}$, and $\mathrm{C}$ in its structure, and it confirms the well-distributed Ni atoms in the whole structure of Ni-ZIF-8. TGA analysis revealed that Ni-ZIF-8 showed better thermal stability from ZIF-8, as it was just a 7\% decreased in weight from 30 to $580^{\circ} \mathrm{C}$, which was probably due to the removal of residual solvent in it (Figure S5). Above $580^{\circ} \mathrm{C}$, there was the breaking of the imidazole molecule in the Ni-ZIF- 8 structure. Furthermore, the FTIR 
analysis (Figure 1 (c)) showed that there was no chemical change occurred in MOF cluster by adding the Ni particles in the ZIF-8

WAXRD analysis was used for testing the microstructure of Ni-ZIF-8 nanoparticles in the MMMs and polymer matrix. As shown in Figure 1(d), there are five main peaks at $12^{\circ}, 18.4^{\circ}, 20.1^{\circ}, 22.9^{\circ}$, and $26.8^{\circ}$ in the pure PDMS/PVDF membrane. These broad peaks confirmed the d-spacing of $7.3 \AA, 4.8 \AA, 4.2 \AA, 3.9$ and $3.3 \AA$, respectively, which can be attributed to the average distance between PDMS and PVDF chains in the membrane. As the Ni-ZIF-8 incorporated with the polymer, PDMS peaks became weak concerning the filler ratio. At $15 \%$ filling of Ni-ZIF-8, these peaks almost minimized as compared with the pure membrane peaks. Such findings were related to a disruption in the chain packing of the polymer due to the addition of Ni-ZIF-8 nanoparticles. In comparison, the major peaks of the Ni-ZIF-8 are conspicuous at high loading and at the same locations for the pure Ni-ZIF-8 peaks, which revealed that no structural changes have occurred in the Ni-ZIF-8 since impregnated with the PDMS.

The FTIR analysis was conducted and shown in Figure 1(e) to check the chemical structures of pure PDMS and Ni-ZIF-8/PDMS MMMs. The pure PDMS membrane and all MMMs spectrums were well matched with the reported data ${ }^{45}$. For pure PDMS membrane, the characteristics peaks were at 1256, 1009, and 788 , which were accredited to the $\mathrm{C}-\mathrm{H}$ symmetric bending vibration, Si-O-Si stretching vibration, and $\mathrm{CH}_{3}$ rocking vibration in $\mathrm{Si}-\mathrm{CH}_{3}$, respectively. These results confirmed the presence of PDMS in the membrane. New bands were found in MMMs at 1310, 1145, and 750, which were attributed to the stretching vibration of $\mathrm{C}-\mathrm{N}$, bending vibration of $\mathrm{C}-\mathrm{H}$, and bending vibration of the imidazole ring, respectively. These new peaks have confirmed the presence of Ni-ZIF- 8 in the polymer matrix. All these peaks confirmed the good affinity of Ni-ZIF-8 nanoparticles with the PDMS and successful formation of MMMs with different Ni-ZIF-8 loadings.

The thermal stabilities of MMMs were observed by using TGA analysis. The results are shown in Figure 1 (f), which showed that pure PDMS has good thermal stability up to $400^{\circ} \mathrm{C}$ and pure Ni-ZIF-8 up to $580^{\circ} \mathrm{C}$. It has been shown that the incorporation of Ni-ZIF-8 particles into the PDMS matrix enhanced the thermal stability of MMMs. Throughout the course of weight loss in the $15 \%$ Ni-ZIF-8 MMM, three steps were found. In the primary step $\left(25-330^{\circ} \mathrm{C}\right)$, there was just $4 \%$ weight loss was observed, which was due to the residual solvent removal from the membranes. While in the second step $\left(330-530^{\circ} \mathrm{C}\right)$, the pyrolysis of PDMS branches occurred, which lost $34.5 \%$ weight of membrane, and in the last step $\left(530-800^{\circ} \mathrm{C}\right) 40 \%$ weight of the membrane was lost due to the degradation of PDMS and Ni-ZIF-8. Except for observing the thermal stabilities of MMMs, the exact loading percentages of the Ni-ZIF-8 were also determined using the yield of the char at the end of this test. The Ni-ZIF-8 loadings from TGA analysis were observed $4.98 \%$, $9.98 \%, 15 \%$, and $20 \%$ for 5-20\% Ni-ZIF-8 loaded MMMs, respectively, which are consistent with the casting solution formulation data of the membranes. 

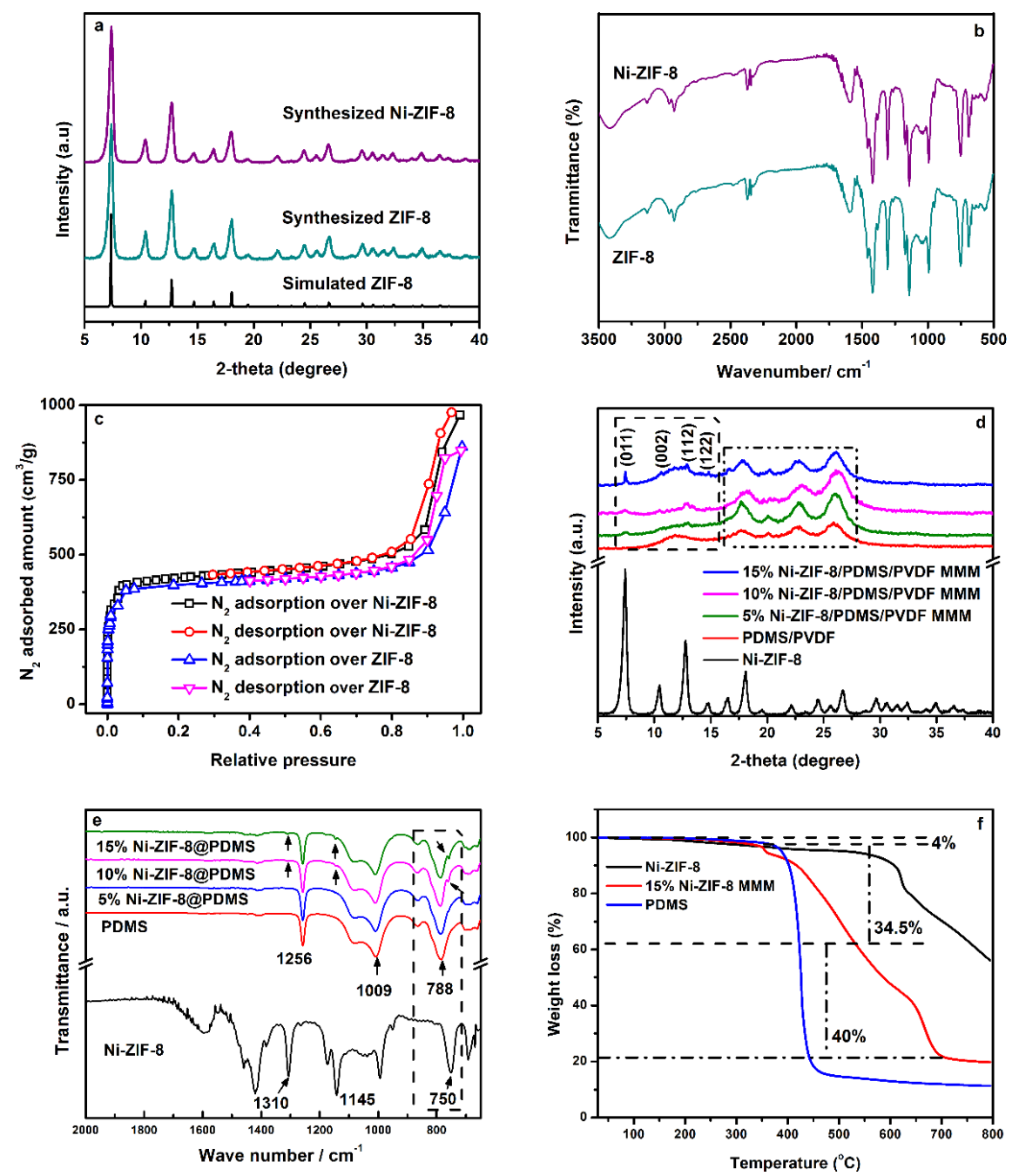

Figure 1. PXRD, $\mathrm{N}_{2}$ adsorption and desorption, and SEM image of Ni-ZIF-8 (a), (b), and (c), while (d), (e), and (f) showing the WXRD, FTIR, and TGA analysis of Ni-ZIF-8 MMMs.

The DSC test will observe the effect of ZIFs on polymer melting and glass transition temperatures, as well as help us to estimate the polymer-MOF interfacial interaction ${ }^{46}$. Table S3 representing the $T_{\mathrm{g}}$ (glass transition temperature), $T_{\mathrm{m}}$ (melting temperature), and $T_{\mathrm{c}}$ (crystallization temperature) of pure PDMS and MMMs. With the increase in Ni-ZIF-8 loading, the DSC results showed the increasing trends in the crystallization and melting temperatures of MMMs as compared with the pure PDMS. The changes in melting and crystallization temperatures were attributed to the reduction of chain mobility of PDMS due to the addition of Ni-ZIF-8 nanoparticles ${ }^{47}$. The PDMS chain mobility reduction may cause the rigid packings of polymer chains, which has shown excellent interfacial interaction between Ni-ZIF-8 and PDMS phases.

SEM images were used at low and higher magnification to check the surface morphologies of the synthesized membranes, as shown in Figure 2. The PDMS control membrane surface was defect-free, dense, and homogeneous (Figure $2 \mathrm{a}$ and b). In comparison, Ni-ZIF-8 nanoparticles observed on the membrane surface, which were well dispersed within the PDMS matrix, implying their good compatibility. The incorporation of Ni-ZIF-8 nanoparticles has resulted in some surface roughness of the MMMs and has increased with respect to the loadings of Ni-ZIF-8. The membrane showed good homogeneity even at high loading up to 15 wt\% of Ni-ZIF-8 (Figure 2 (c) and (d)). It was observed that the nanoparticles of Ni-ZIF-8 started to agglomerate 
marginally at a loading of $20 \mathrm{wt} \%$ (Figure S6 and S7), which may be due to the stronger surface tension of ZIF particles than to the polymer affinity. The overall well scattered ZIFs in the polymer is $15 \mathrm{wt} \%$ loadings. At lower magnification, the nanoparticles of Ni-ZIF-8 tend to be aggregated, but at a higher magnification, they are very well dispersed from each other, and no defects were observed in the MMMs. Cross-sectional morphologies of the 15\% Ni-ZIF-8 MMM have been shown in Figure 2 (e and f) with low and high magnifications, respectively. There was no sign of voids observed between the membrane active layer and the PVDF support. The average thickness of the $15 \%$ Ni-ZIF- 8 MMM active layer was $13 \mu \mathrm{m}$. At higher magnification, Ni-ZIF-8 nanoparticles can be seen in the active layer of the membrane within the PDMS.

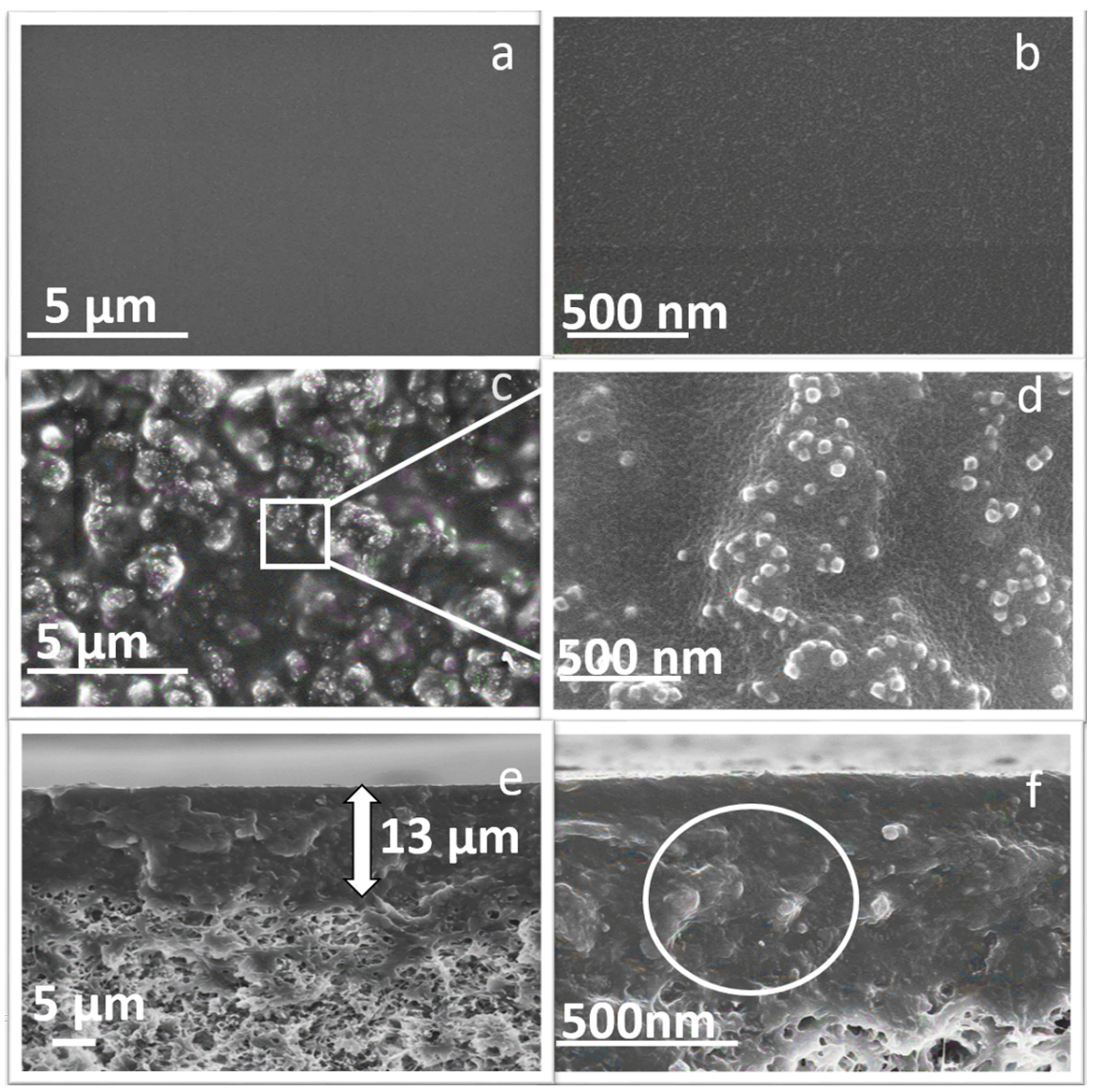

Figure 2. Surface morphology of PDMS (a, b) and 15\% Ni-ZIF-8 MMM (c, d), cross section image of $15 \%$ Ni-ZIF-8 MMM (e, f).

In addition to surface morphologies, the dispersion of Ni-ZIF-8 nanoparticles within the polymer matrix was further verified using the EDX analysis shown in Figure S8. The continuous grey and green colors representing the $\mathrm{Si}$ and $\mathrm{O}$, respectively, reflecting $\mathrm{Si}-\mathrm{O}$ backbone of the polymer matrix. The $\mathrm{Si}$ and $\mathrm{O}$ colors have filled the mapping, which confirmed that all Ni-ZIF-8 nanoparticles are wrapped with the PDMS layer. The blue, red, and yellow colors are representing the $\mathrm{Zn}, \mathrm{N}$, and $\mathrm{Ni}$, respectively. The good distribution of these three colors confirmed the excellent dispersion of Ni-ZIF-8 nanoparticles within the polymer matrix, which might be beneficial to create the relatively continuous pathways for molecular diffusion. In order to improve the separation performance and defect-free membrane synthesis, homogeneous dispersion of the filler, and excellent compatibility between organic and inorganic phases must be created ${ }^{12}$.

The tests of TGA, EDX, SEM, and EDX revealed that the Ni-ZIF-8 has excellent interaction with PDMS chains, and could activate the Van der Waals forces and hydrogen bonding between Ni-ZIF-8 and polymer 
phases. Moreover, the addition of Ni-ZIF-8 in the PDMS did not alter the crystallinity and chemical structure of PDMS, as shown by XRD and FTIR analysis.

\section{Gas separation properties of MMMs}

\section{Effect of ZIFs loading}

Pure gas permeation experiments were conducted at a pressure of 1.5 bar and a temperature of $40^{\circ} \mathrm{C}$, for the evaluation of pure PDMS, and MMMs. Figure 3 demonstrates the permeation of BD in pure PDMS and MMMs with ZIF-8 and Ni-ZIF-8 loadings. It can be observed that the permeation improved by the increase in ZIFs loading in both types of MMMs, while the Ni-ZIF-8 based MMMs showed a significant rise in permeation with respect to the ZIF-8 based MMMs. The highest permeation results were observed at $20 \%$ ZIFs loading, which were 400 GPU for Ni-ZIF-8 based MMM and 325 GPU for ZIF-8, and showed improvements of 98\%, and $61 \%$ as compared with pure PDMS membrane, respectively. The reason for Ni-ZIF-8/PDMS MMMs has more permeation than pure ZIF-8/PDMS MMMs can be described in three different parameters. The first parameter was the large surface area, high micro, and total pore volumes of Ni-ZIF-8 relative to the pure ZIF-8. The second parameter was the tunability of the effective aperture size of the ZIF- 8 by the inclusion of the $\mathrm{Ni}$ atoms in its cluster. Pure ZIF-8 has experimentally demonstrated an effective aperture size of 4.0-4.2 $\AA$ (0.6-0.8 $\AA$ higher than its aperture size $(3.4 \AA)$ showed by XRD analysis $)^{48}$, which is smaller than the kinetic diameter of the BD molecule $(4.3 \AA)$. In addition, the simulation findings have shown that the organic linker in the ZIF-8 is able to fluctuate the opening size up to $1 \AA$, which means that the pure ZIF- 8 will expand its pores up to $4.4 \AA^{49}$. Based on these facts, we assumed the same organic linker fluctuation in Ni-ZIF-8 that enhanced its aperture size by $0.8-1.0 \AA$, and it showed an effective aperture size of 4.4-4.6 $\AA$, which is larger than the size of BD molecule. These evidences proved that the Ni-ZIF- 8 pore/aperture has more compatibility with the size of the $\mathrm{BD}$ molecule than the ZIF-8 pore/aperture, making it more convenient for the BD molecules penetration. The third parameter was the presence of more metal sites ( $\mathrm{Zn}$ and $\mathrm{Ni}$ ) in Ni-ZIF-8 cluster relative to the ZIF-8 (Zn), which provided more affinity to butadiene molecules.

$\mathrm{BD}$ molecule is unsaturated due to two $\mathrm{C}=\mathrm{C}$ double bonds in its structure. This unsaturation gives it the capacity to interact with the metal sites that are normally electron-rich. The BD molecule attracted towards the two-metal sites of Ni-ZIF-8, which have different adsorption and binding behavior based on the $\pi$-complexation mechanism for the BD molecules. Owing to this discrepancy, it gave the asymmetric adsorption/desorption of BD from Ni-ZIF-8, which enhanced the permeation from Ni-ZIF-8/PDMS MMMs. $\mathrm{BD}$ adsorption in the ZIFs based on metal-to-BD molecule interplay polarization that can form a reversible complex with transition metals. Based on previous work ${ }^{50}$, it was postulated that the BD interacted with the $\mathrm{Ni}$ and $\mathrm{Zn}$ metals atomic orbitals, forming a complex. During this complex, BD and metals act as an electron acceptor and electron donor, respectively. By overlapping the metal's external s-orbitals with the BD's bonding $\pi$-molecular orbital, a $\sigma$-bond is formed in the complex. Additionally, a $\pi$-bond formed in the complex due to the transfer of electrons from the BD's vacant antibonding $\pi^{*}$-molecular orbital and filled d-atomic metal orbital. In desorption, the same reverse effect happened, releasing the BD molecule from metal sites. In Ni-ZIF-8, the presence of two metals created the synergistic effect and reinforced BD affinity.

On the other hand, Figure 3 shows the ideal selectivity of pure PDMS membrane and MMMs for BD/ $\mathrm{N}_{2}$ separation with respect to both types of ZIFs loadings. It can be found that the selectivity improved by increasing the ZIFs loading in the PDMS matrix up to $15 \%$, and thereafter the selectivity decreased in both types of MMMs. The contradiction between permeance and selectivity at $20 \%$ ZIF loading is the so-called "trade-off effect" of polymeric membranes. Compared to the pure PDMS membrane, the Ni-ZIF-8 displayed an overall improvement in selectivity about $81 \%$, while the ZIF- 8 showed $32 \%$ at the same (15\%) loadings. Thus, ZIFs with a load of not more than $15 \%$ broke the trade-off law between permeability and selectivity. The reduction of selectivity at higher loading may be due to internal interface defects in the agglomeration of the ZIFs within the PDMS matrix. Ni-ZIF-8/MMMs has more selectivity and permeation than the ZIF$8 / \mathrm{MMMs}$, which can be explained by the high affinity of Ni-ZIF- 8 with BD, and low interaction with the nitrogen molecules. It was also observed that the nitrogen permeance was slightly higher in ZIF-8 based MMMs than the Ni-ZIF-8/MMMs, which also affected the selectivity of ZIF-8/MMMs. 
Based on the aforementioned results of permselectivity, $15 \%$ of Ni-ZIF-8 MMM were selected for further evaluation under different pressure and temperature conditions. Improved permeation properties, particularly $81 \%$ improvement in $\mathrm{BD} / \mathrm{N}_{2}$ ideal selectivity, strongly confirmed that the synthesized Ni-ZIF-8/MMMs up to $15 \mathrm{wt} \%$ loadings are defect-free. For the $99 \%$ hydrocarbons recovery from nitrogen mixture, rubbery membranes with 100 GPU permeability and 10 selectivity can be used with the proper design of the membrane process ${ }^{29}$. Therefore, it is believed to use $15 \%$ Ni-ZIF- 8 MMMs made in this study, having 323 GPU permeance and 19.5 selectivity for efficient separation of BD from the $\mathrm{N}_{2}$ mixture.
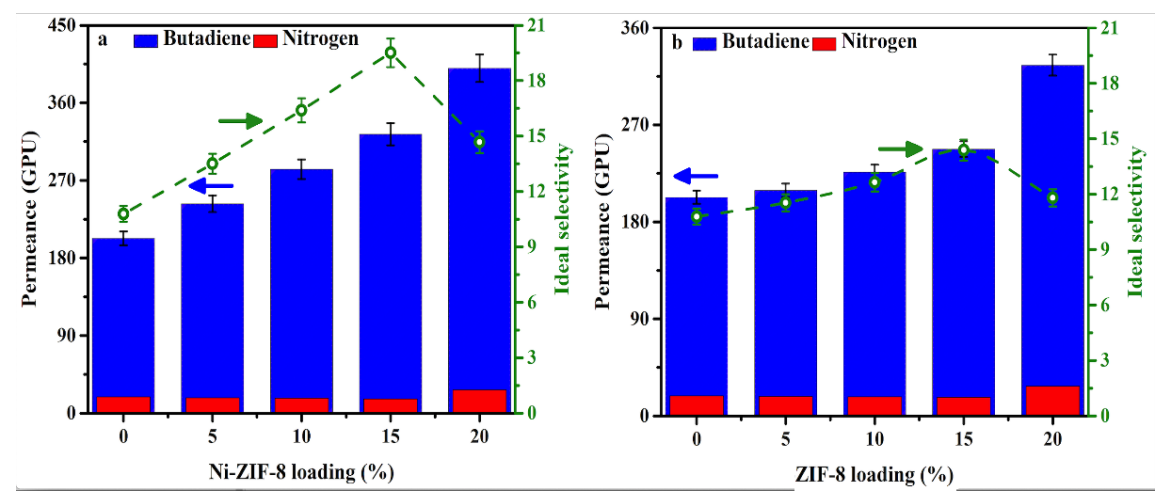

Figure 3. Gas separation properties with respect to ZIF loadings of (a) Ni-ZIF-8 MMMs and (b) ZIF-8 MMMs

\section{Effect of feed temperature and pressure}

The membranes were tested at different operating temperatures and a constant feed pressure of 1.5 bar. Figure 4 ( $\mathrm{a}$ and $\mathrm{b}$ ) showing the effect of temperature on the permeation and ideal selectivity $\left(\mathrm{BD} / \mathrm{N}_{2}\right)$ in pure PDMS and Ni-ZIF-8 MMMs. The temperature range was set according to the actual emission conditions of synthetic rubber plant tail gas. The permeance of butadiene decreased in pure PDMS and 15\% Ni-ZIF-8 MMM with respect to the increase in operating temperature, which confirmed that the solubility phenomenon is dominant in the transportation of butadiene across the membranes. The BD permeance decreased from 323 to $284 \mathrm{GPU}$ in $15 \% \mathrm{Ni}$-ZIF-8 MMM from 40 to $50^{\circ} \mathrm{C}$ temperature increase. While the permeance in pure PDMS was reduced from 202 to $187 \mathrm{GPU}$ at the same temperature change. It was worth noticing that the effect of temperature on Ni-ZIF-8/PDMS MMMs permeance is more prominent compared with pure PDMS, which can be explained due to the less adsorption of BD over Ni-ZIF-8 at high temperature as shown in Figure S10, and the same trend existed in its MMMs.

In the case of nitrogen, the permeance enhanced at elevated temperature, which suggested that the diffusion phenomenon is responsible for the transport of nitrogen gas molecules through the membranes. In this work, the nitrogen permeance improved from 18.8 to 19.6 GPU in PDMS and from 16.6 to 17.9 GPU in $15 \%$ Ni-ZIF-8 MMM, respectively, by rising the temperature from 40 to $50^{\circ} \mathrm{C}$. The $\mathrm{BD} / \mathrm{N}_{2}$ ideal selectivities decreased at elevated temperatures; the pure PDMS selectivity decreased 12.5\%, while15\% Ni-ZIF-8 MMM selectivity decreased $20 \%$ from 40 to $50^{\circ} \mathrm{C}$, respectively. The decrement of selectivity was higher in Ni-ZIF- 8 MMM, which could be ascribed to the low sorption of BD at high temperatures. The low selectivity at high temperature indicates that the temperature should be maintained at $40^{\circ} \mathrm{C}$ for further membranes testing. From the previously reported works [26,39], it was concluded that the separation of the hydrocarbons through the gas separation system was affected by the high temperature, so that the low operating temperature favored the recovery of hydrocarbons from the mixed nitrogen stream. Consequently, $40^{\circ} \mathrm{C}$ temperature was selected for further testing of these membranes.

The effect of feed pressure was tested on pure PDMS, and all Ni-ZIF-8/MMMs at a constant temperature of $313 \mathrm{~K}$. Figure $4(\mathrm{c}, \mathrm{d})$ presenting the effect of pressure on separation properties of membranes, in which the 
permeance enhanced with respect to the feed pressure. By increasing the pressure from 1.5 to 2.5 bar, the BD permeance improved by $10 \%$ in pure PDMS and improved by $14 \%$ in $15 \%$ Ni-ZIF- 8 MMM, respectively. Nitrogen permeance increased from 18.8 GPU to 22 GPU in pure PDMS, and 16.6 GPU to 20 GPU in $15 \%$ Ni-ZIF-8 MMM, respectively. The increase in permeability was mainly due to the high trans-membrane pressure, which is the driving force of the gas molecules transportation across the membrane.

The ideal selectivity has decreased slightly (from 10.78 to 10.15 ) in pure PDMS, and from 19.5 to 18.3 in $15 \%$ Ni-ZIF-8 MMM by increasing the feed pressure from 1.5 to 2.5 bar, respectively. The reduction of selectivity was mainly due to the increment in nitrogen permeance by elevating the feed pressure. The details about the permeation mechanism, which includes the sorption and diffusion of both gases, will be discussed in a later section. As the performance of the membrane considered better at low transmembrane pressure, so that 1.5 bar feed pressure was selected for the further analysis of these membranes.
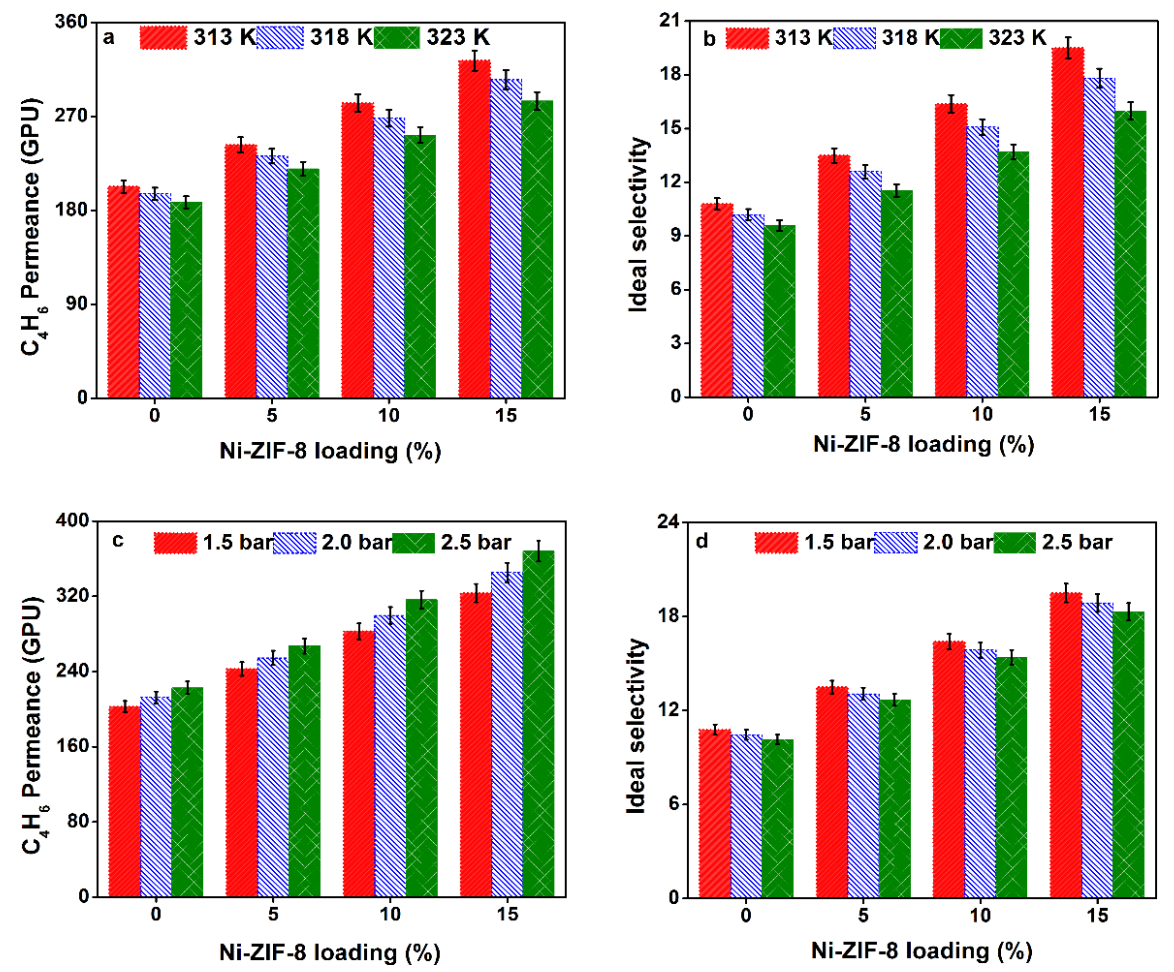

Figure 4. Effect of operating temperature (a), (b) and pressure (c), (d) on gas separation properties of pure PDMS membrane, and Ni-ZIF-8 MMMs.

\section{Sorption and diffusion analysis}

For a better understanding of permeation mechanism across the pure PDMS membrane and MMMs, sorption tests were conducted at different temperatures, and a constant pressure range from 0-1.5 bar. As the gas separation in rubbery polymers follows the solution-diffusion model so that both the sorption and diffusion properties of BD and $\mathrm{N}_{2}$ have been measured for pure PDMS, ZIF-8/PDMS MMMs, and Ni-ZIF-8/PDMS MMMs. The isotherm of BD sorption in PDMS is linear, while the ZIF-8 and Ni-ZIF-8 based MMMs showed a slight curve with respect to the ZIFs filling in the membrane (Figure 5 (a) and Figure S11). The concentration of $\mathrm{BD}$ in the membrane increased with respect to the loading of the ZIFs particles in the PDMS matrix. The details are given in supplementary information.

The solution coefficients of both gases and solution selectivity are determined by using equations (5) and (6), as shown in Figure 5 (b) and Figure S14(a). The BD solution coefficient enhanced from 24.67 to 35.13 
$\left(\mathrm{cm}^{3}\right.$ (STP). $\mathrm{cm}^{-3}$ membrane. bar-1) by incorporating Ni-ZIF-8 particles up to $15 \%$ in the PDMS matrix, which is quite similar to the calculated theoretical sorption values of MMMs by using pure Ni-ZIF-8 and PDMS adsorption capacity data (supporting information Table S11). Consistency of experimental and theoretical solution coefficient values verified that no pore blockage of the fillers existed due to polymer chains in the MMM synthesis. The maximum solution selectivity measured was 27.43 for $15 \%$ Ni-ZIF- 8 MMM at $40^{\circ} \mathrm{C}$ and 1.5 bar pressure, which was $31 \%$ improved by PDMS, and $18 \%$ higher than $15 \%$ ZIF- 8 MMM value. This phenomenon should be ascribed to the intrinsically high adsorption capacity and adsorption selectivity of Ni-ZIF-8 than those of the ZIF-8, and PDMS. The rise in solubility due to the Ni-ZIF-8 filling, possibly due to the decrease in polymer density or high fractional free volume of the $\mathrm{MMMs}^{51}$.

The penetrant solubility in the polymer matrix mainly depends upon two factors. One factor is dependent, and the other is independent of polymer-penetrant interaction ${ }^{52}$. The values of Hansen solubility parameters of PDMS and BD are given in Table S10, which indicates the difference of 0.94 between BD and PDMS. The lower difference in solubility parameters shows higher polymer-penetrant interaction. The other parameter that influences the penetrant solubility is its critical temperature $\left(T_{c}\right)$, which is used to calculate the penetrant condensability. Consequently, the penetrant, which has a high value of $T_{\mathrm{c}}$, shows high condensation and high solubility. The critical temperature of $\mathrm{BD}$ is $425 \mathrm{~K}$ (Table S5), which is much higher than the $\mathrm{N}_{2}$ critical temperature $(126 \mathrm{~K})$. According to these considerations, BD solubility is higher in PDMS membrane than $\mathrm{N}_{2}$, while the introduction of ZIF-8 and Ni-ZIF-8 also affects the solubility of both the penetrants in MMMs.

Molecular diffusivity is positively correlated with diffusion coefficient $(D)$ and concentration difference driving force. The diffusion coefficient and diffusion selectivity $\left(a_{\mathrm{D}}\right)$ data are given in Figure 5(c) and Figure S14. The diffusivity of $15 \%$ Ni-ZIF-8/MMM $45 \%$ improved by pure PDMS, and $18 \%$ higher than $15 \%$ ZIF8/MMM. The increase in diffusivity with respect to Ni-ZIF-8 loading was due to an increase in the fractional free volume of the membrane, and the nano-sized particles enhanced the polymer-filler interface, which provided the efficient gas diffusion channels as compared with pure PDMS, and ZIF-8/MMMs. The diffusion selectivity also improved with respect to the ZIFs loading in the PDMS matrix. Diffusion selectivity of $15 \%$ Ni-ZIF-8 MMM showed 39\% improvement from pure PDMS, and 16\% from 15\% ZIF-8 MMM, respectively. The diffusional selectivity has been improved mainly due to the provided channels of Ni-ZIF-8 cavities to BD molecules and less interaction of $\mathrm{N}_{2}$ with respect to the Ni-ZIF-8 loadings. As the ideal selectivity is the multiplication of solution and diffusion selectivities, so that the contribution of diffusion selectivity (0.7) of $15 \%$ Ni-ZIF-8 MMM to the ideal selectivity (19.5) is low compared to the solution selectivity (27.4), shown in Figure 5(d).

In order to explain the diffusion of penetrants briefly, the effective diameter of a gas molecule for diffusion in the membrane should be known. For this purpose, either collision diameter of Lennard-Jones potential $\sigma$ LJ or kinetic diameter $a_{\mathrm{kt}}$ given by Breck often been used $^{53}$. The effective diameter of BD estimated at 0.43 $\mathrm{nm}$ ( supporting information), which is smaller than its Leonard Jones diameter $(0.51 \mathrm{~nm})^{54}$, but similar to its cross-section diameter $(0.44 \mathrm{~nm})$ of the molecule. Therefore, it was estimated that the diffusion of BD molecules through the membrane was based on its kinetic molecule diameter. 

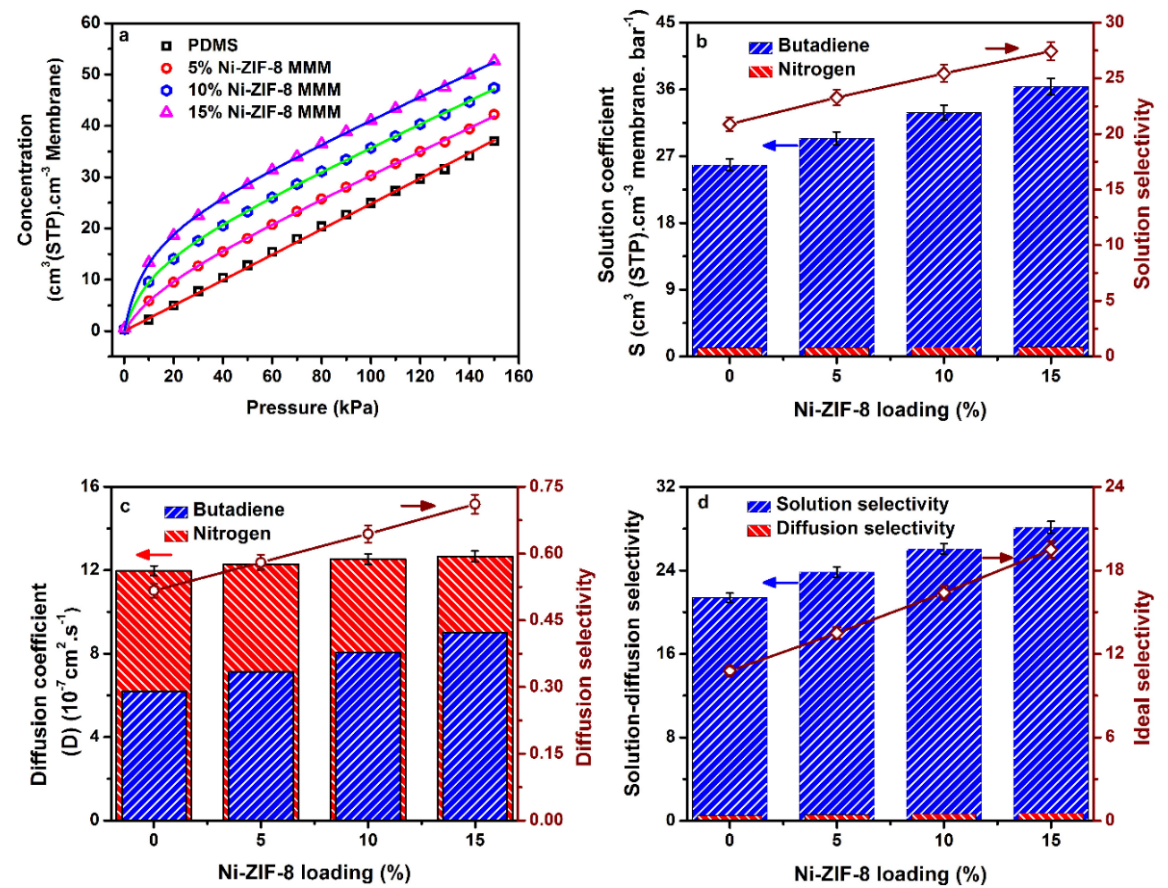

Figure 5. Effect of Ni-ZIF-8 loading over concentration of BD (a), solution coefficient of BD and solution selectivity (b), diffusion coefficient and diffusion selectivity (c), and contribution of solution selectivity and diffusion selectivity in ideal $\mathrm{BD} / \mathrm{N}_{2}$ selectivity(d).

\section{Effect of temperature on solubility and diffusivity}

Effect of temperature was also tested for both penetrant solubilities and diffusivities in pure PDMS, ZIF8, and Ni-ZIF-8 based MMMs. Figure 6(a, b), and Figure S15 (a, b) show the solution coefficients and solution selectivities of BD with respect to the feed temperatures over Ni-ZIF-8 MMMs, and ZIF-8 MMMs, respectively. It was noticed that the solution coefficient of both the penetrants was decreased with respect to the feed temperatures. The BD solution coefficient values decreased from 35.13 to 26.5 in $15 \%$ in Ni-ZIF- 8 MMM, from 31.9 to 24.12 in $15 \%$ ZIF-8 MMM, and from 24.67 to $19\left(\mathrm{~cm}^{3}\right.$ (STP).cm ${ }^{-3}$ membrane. bar $\left.{ }^{-1}\right)$ in PDMS from 40 to $50^{\circ} \mathrm{C}$, respectively. These findings indicate the sorption domination in $\mathrm{BD}$ permeation across the membrane, as the BD permeance and ideal selectivity also decreased at elevated temperatures.

The diffusion coefficient values of $\mathrm{BD}$ and diffusion selectivity increase with respect to feed temperatures shown in Figure 6 (c, d), and Figure S15 (c, d) for Ni-ZIF-8 MMMs, and ZIF-8 MMMs, respectively. In rubbery polymers such as PDMS, the diffusion coefficient normally increased by the increase in temperature. BD diffusion coefficient increased $19 \%$ in pure PDMS, $16 \%$ in 15\% ZIF-8 MMM, and $17 \%$ in 15\% Ni-ZIF-8 MMM, respectively. On the other side, the diffusion selectivity also increased with respect to the increase in temperature. It was noted that the selectivity enhancement was slightly more (11\%) in pure PDMS as compared with 15\% ZIF-8 MMM (9\%), and 15\% Ni-ZIF-8 MMM (8.5\%), which can be attributed to decreased BD sorption at elevated temperatures in ZIF-8 and Ni-ZIF-8. 

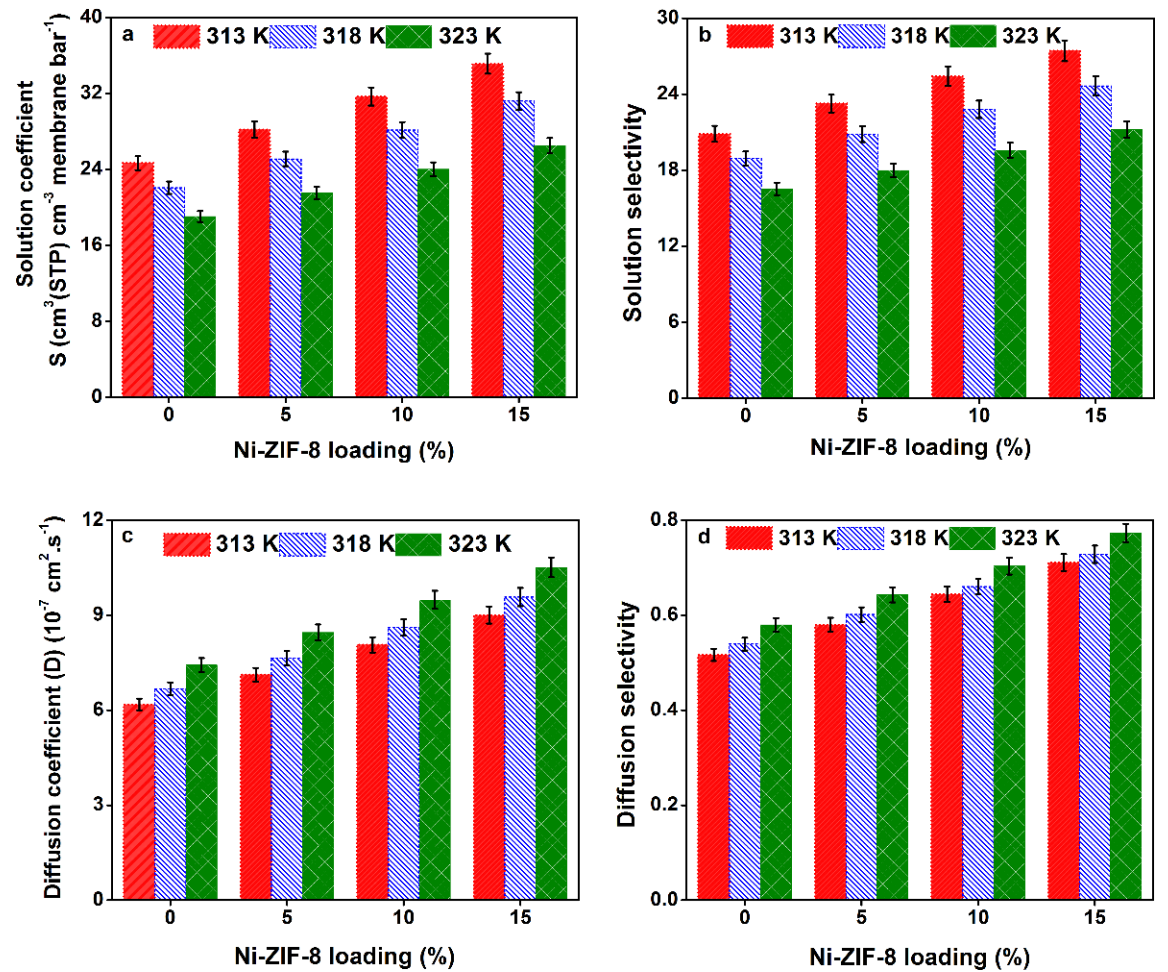

Figure 6. Effect of temperature on BD solution coefficient (a), solution selectivity (b), diffusion coefficient (c), and diffusion selectivity (d) in pure PDMS, and Ni-ZIF-8 MMMs.

\section{Permeation activation energies}

For finding the effects of temperature over permeability, gas sorption, and gas diffusion, the permeation activation energies were calculated for detecting the contribution of each parameter in the permeation of penetrants through the membrane. Permeation activation energy provides a qualitative analysis for testing the permeation mechanism. The temperature dependency of gas sorption, diffusion, and permeability can be described by using the following Arrhenius-Van't Hoff equations:

$$
\begin{aligned}
& S=S_{0} \exp \left(-\frac{H_{S}}{\mathrm{RT}}\right) \\
& D=D_{0} \exp \left(-\frac{E_{D}}{\mathrm{RT}}\right) \\
& P=P_{0} \exp \left(-\frac{E_{p}}{\mathrm{RT}}\right)
\end{aligned}
$$

here $S_{0}, D_{0}$, and $P_{0}$ representing pre-exponential factors of sorption, diffusion, and permeation, respectively. $R$ is used for general gas constant $\left(8.314 \mathrm{~kJ} \mathrm{~mol}^{-1} . \mathrm{K}\right)$, and $T$ is the operating temperature $(\mathrm{K}) . \Delta H_{\mathrm{S}}, E_{\mathrm{D}}$, and $E_{\mathrm{P}}$ are representing the enthalpy of sorption, the apparent activation energy of diffusion, and apparent activation energy of permeability, respectively.

Penetrant sorption in the membrane may be considered as a two-step thermodynamic process: (1) penetrant condensation from a gas phase density to a liquid-like density and (2) opening a gap in the membrane to allow the condensed penetrant to be mixed with the active membrane layer. In order to better understand the role of each thermodynamic phase in the determination of penetrant solubility, these two factors are dealt with separately. The condensation step value was directly measured by reversing the penetrant heat of vaporization, while the other step value was measured by using the following equation,

$H_{S}=H_{\text {cond }}+H_{\text {mix }}$ 
here the $H_{\mathrm{S}}$ is the enthalpy change of sorption, $\Delta H$ cond is the heat of condensation of the penetrant, and $\Delta H_{\text {mix }}$ is the heat of mixing, which is required for the mixing of gas molecules with the membrane surface.

The equation (12) was used for the qualitative dissolution analysis of penetrants in the membrane. It was noticed that the low mixing heat demonstrated a strong penetrant affinity to the membrane ${ }^{55}$. The lower mixing value of $15 \%$ Ni-ZIF-8 MMM confirmed the higher BD affinity with this membrane relative to pure PDMS, and 15\% ZIF-8 MMM. The values are given in Table 1, which shows that $\Delta H_{\mathrm{S}}$ has negative values for both the gases as the solubility decreased by temperature increase. On the other hand, the $\mathrm{N}_{2}$ showed a positive mixing heat in pure PDMS, 15\% ZIF- 8 MMM and 15\% Ni-ZIF-8 MMM. The positive mixing heat showed less affinity of $\mathrm{N}_{2}$ with pure PDMS while mixing heat increased by $28 \%$ in $15 \%$ ZIF- 8 MMM and $67 \%$ in $15 \%$ Ni-ZIF-8 MMM. The high mixing heat value strongly supports the low $\mathrm{N}_{2}$ interaction with $15 \%$ Ni-ZIF-8 MMM.

Table 1. Energy analysis for penetrant permeation through PDMS, 15\% ZIF-8 MMM, and 15\% Ni-ZIF-8 MMM.

\begin{tabular}{|c|c|c|c|}
\hline Activation energies & Pure PDMS membrane & 15\% ZIF-8 MMM & 15\% Ni-ZIF-8 MMM \\
\hline$E_{\mathrm{P}}$ of $\mathrm{BD}\left(\mathrm{kJ} \cdot \mathrm{mol}^{-1}\right)$ & -6.40 & -8.41 & -10.67 \\
\hline$E_{\mathrm{P}}$ of $\mathrm{N}_{2}\left(\mathrm{~kJ} \cdot \mathrm{mol}^{-1}\right)$ & +3.5 & +4.2 & +5.0 \\
\hline$E_{\mathrm{D}}$ of $\mathrm{BD}\left(\mathrm{kJ} \cdot \mathrm{mol}^{-1}\right)$ & +15.38 & +13.81 & +12.98 \\
\hline$E_{\mathrm{D}}$ of $\mathrm{N} 2\left(\mathrm{~kJ} . \mathrm{mol}^{-1}\right)$ & +5.7 & +6.2 & +6.8 \\
\hline$H_{\mathrm{S}}$ of $\mathrm{BD}\left(\mathrm{kJ} \cdot \mathrm{mol}^{-1}\right)$ & -21.79 & -22.32 & -23.67 \\
\hline$H_{\mathrm{S}}$ of $\mathrm{N}_{2}\left(\mathrm{~kJ} \cdot \mathrm{mol}^{-1}\right)$ & -2.2 & -2.03 & -1.8 \\
\hline$H_{\text {cond }}$ of $\mathrm{BD}\left(\mathrm{kJ} \cdot \mathrm{mol}^{-1}\right)$ & $-20.86^{56}$ & $-20.86^{56}$ & $-20.86^{56}$ \\
\hline$H_{\text {cond }}$ of $\mathrm{N}_{2}\left(\mathrm{~kJ} . \mathrm{mol}^{-1}\right)$ & $-2.8^{*}$ & $-2.8^{*}$ & $-2.8^{*}$ \\
\hline$H_{\text {mix }}$ of $\mathrm{BD}\left(\mathrm{kJ} . \mathrm{mol}^{-1}\right)$ & -0.9 & -1.46 & -2.8 \\
\hline$H_{\text {mix }}$ of $\mathrm{N}_{2}\left(\mathrm{~kJ} \cdot \mathrm{mol}^{-1}\right)$ & +0.6 & +0.77 & +1.0 \\
\hline
\end{tabular}

${ }^{*}$ Condensation heat was evaluated at $40^{\circ} \mathrm{C}$

Form the previously reported data ${ }^{57}$, it was noticed that the $\ln \left(D_{0}\right)$ is proportional to the activation entropy change. Thus, the decreased diffusional activation energy in 15\% Ni-ZIF-8 MMM confirms that the activation entropy change is less in the 15\% Ni-ZIF-8 MMM relative to the pure PDMS, and $15 \%$ ZIF-8 MMM. Besides, Barrer's "zone" theory of diffusion also states that a penetrant molecule executes a diffusion jump in an activated zone comprising segments of polymer molecules near the diffusing penetrant molecule. So, the size of the activated zone is also associated with both $E$ D and activation entropy $(\ln D$ $\left.{ }_{0}\right)$. Consequently, the larger the size of the activated zone required for diffusion, the higher is the diffusional activation energy and the entropy change, which is associated with the creation of the activated zone ${ }^{58}$. Therefore, it was concluded that the addition of Ni-ZIF-8 in PDMS reduced the size of activated zones for BD molecule jumps for diffusion, and also reduced the entropy change. Moreover, the permeation activation energy of BD reduced $66 \%$ in 15\% Ni-ZIF-8 MMM from PDMS, and $27 \%$ less than $15 \%$ ZIF-8 MMM. Contrary, the permeation activation energy of $\mathrm{N}_{2}$ in $15 \%$ Ni-ZIF- 8 MMM showed a $43 \%$ increased from PDMS and 19\% higher than 15\% ZIF-8 MMM.

\section{Conclusions}

In this study, ZIF-8 and Ni-ZIF-8 nanoparticles were incorporated with PDMS for the synthesis of MMMs, and the separation mechanisms of butadiene and nitrogen, molecular solution-diffusion properties through the MMMs, were investigated comprehensively.

Ni-ZIF-8 nanoparticles have been used for the first time with PDMS to synthesize MMMs. The resulting Ni-ZIF-8/PDMS MMMs significantly improved thermal stability, microstructure, permeance, and selectivity relative to pure PDMS, and ZIF-8/PDMS MMMs. Interestingly, Ni-ZIF-8 MMM significantly improved BD 
permeance and selectivity at the same time, breaking down the trade-off effect. Ni-ZIF-8/PDMS MMMs showed greater BD affinity relative to ZIF-8/PDMS MMMs under the same experimental conditions. BD permeance in $15 \%$ Ni-ZIF- 8 was improved by $67 \%$ from PDMS, and $32 \%$ from $15 \%$ ZIF-8 MMM. The ideal selectivity of $\mathrm{BD} / \mathrm{N}_{2}$ of the corresponding $15 \%$ Ni-ZIF- 8 MMM was increased by $81 \%$ and $37 \%$ from pure PDMS and 15\% ZIF-8/MMM, respectively. The higher BD/ $\mathrm{N}_{2}$ ideal selectivity of Ni-ZIF-8/PDMS over that of ZIF-8/PDMS was attributed to the effective aperture size and high affinity of Ni-ZIF-8 with butadiene.

The solution-diffusion results revealed in detail for these mixed-matrix membranes. The solubility, diffusivity, solubility selectivity, and diffusion selectivity were improved in Ni-ZIF-8@PDMS MMMs. Permeation activation energies revealed that the $15 \%$ Ni-ZIF-8 MMM showed less activation energy of diffusion, the activation energy of permeation, activation entropy change, and sorption enthalpy relative to pure PDMS, and 15\% ZIF-8 MMM. All of these findings strongly support the use of 15\% Ni-ZIF-8 MMM for the efficient separation of BD and nitrogen, which is available in the tail gas of the synthetic rubber industry. This study will provide in-depth knowledge of gas transport properties in ZIFs-based MMMs for the separation of VOCs from permanent gases.

\section{Acknowledgments}

This study was financially supported by the National Natural Science Foundation of China (No. 21736001). We also acknowledge the research support from the Analysis \& Testing Center of Beijing Institute of Technology.

\section{References}

1. Jeffrey S. Plotkin. The Continuing Quest for Butadiene - American Chemical Society. Cutting-Edge Chemistry. https://www.acs.org/content/acs/en/pressroom/cutting-edge-chemistry/the-continuing-questfor-butadiene.html. Published 2016. Accessed September 8, 2019.

2. Chemical Economics Handbook (CEH) . IHS Markit https://ihsmarkit.com/products/butadiene-chemicaleconomics-handbook.html. Accessed April 19, 2019.

3. Doyle M, Sexton KG, Jeffries H, Bridge K, Jaspers I. Effects of 1,3-butadiene, isoprene, and their photochemical degradation products on human lung cells. Environ Health Perspect . 2004;112(15):14881495. doi:10.1289/ehp.7022

4. Liu G, Chernikova V, Liu Y, et al. Mixed matrix formulations with MOF molecular sieving for key energy-intensive separations. Nat Mater . 2018;17(3):283-289. doi:10.1038/s41563-017-0013-1

5. Wang J, Fang W, Luo J, et al. Selective separation of CO2 using novel mixed matrix membranes based on Pebax and liquid-like nanoparticle organic hybrid materials. J Memb Sci . 2019;584:79-88. doi:10.1016/j.memsci.2019.04.079

6. Zhao J, Xie K, Liu L, Liu M, Qiu W, Webley PA. Enhancing plasticization-resistance of mixed-matrix membranes with exceptionally high $\mathrm{CO} 2 / \mathrm{CH} 4$ selectivity through incorporating ZSM-25 zeolite. J Memb Sci . 2019;583:23-30. doi:10.1016/j.memsci.2019.03.073

7. Oh JW, Cho KY, Kan MY, Yu HJ, Kang DY, Lee JS. High-flux mixed matrix membranes containing bimetallic zeolitic imidazole framework-8 for C3H6/C3H8 separation. J Memb Sci . 2020;596. doi:10.1016/j.memsci.2019.117735

8. Li S, Li P, Cai D, et al. Boosting pervaporation performance by promoting organic permeability and simultaneously inhibiting water transport via blending PDMS with COF-300. J Memb Sci . 2019;579:141150. doi:10.1016/j.memsci.2019.02.041

9. Hu J, Cai H, Ren H, et al. Mixed-matrix membrane hollow fibers of Cu3(BTC)2 MOF and polyimide for gas separation and adsorption. Ind Eng Chem Res . 2010;49(24):12605-12612. doi:10.1021/ie1014958 
10. Kitao T, Zhang Y, Kitagawa S, Wang B, Uemura T. Hybridization of MOFs and polymers. Chem Soc Rev . 2017;46(11):3108-3133. doi:10.1039/c7cs00041c

11. Moore TT, Koros WJ. Non-ideal effects in organic-inorganic materials for gas separation membranes. $J$ Mol Struct . 2005;739(1-3):87-98. doi:10.1016/j.molstruc.2004.05.043

12. Venna SR, Lartey M, Li T, et al. Fabrication of MMMs with improved gas separation properties using externally-functionalized MOF particles. J Mater Chem A . 2015;3(9):5014-5022. doi:10.1039/c4ta05225k

13. Mao H, Li SH, Zhang AS, Xu LH, Lu JJ, Zhao ZP. Novel MOF-capped halloysite nanotubes/PDMS mixed matrix membranes for enhanced n-butanol permselective pervaporation. J Memb Sci . February 2019. doi:10.1016/j.memsci.2019.117543

14. Xu S, Zhang H, Yu F, Zhao X, Wang Y. Enhanced ethanol recovery of PDMS mixed matrix membranes with hydrophobically modified ZIF-90.Sep Purif Technol . 2018;206:80-89. doi:10.1016/j.seppur.2018.05.056

15. Şahin F, Topuz B, Kalıpçılar H. ZIF filled PDMS mixed matrix membranes for separation of solvent vapors from nitrogen. J Memb Sci . 2020;598:117792. doi:10.1016/j.memsci.2019.117792

16. Meshkat S, Kaliaguine S, Rodrigue D. Mixed matrix membranes based on amine and nonamine MIL-53(Al) in Pebax@ MH-1657 for CO2 separation.Sep Purif Technol . 2018;200:177-190. doi:10.1016/j.seppur.2018.02.038

17. Prasetya N, Teck AA, Ladewig BP. Matrimid-JUC-62 and Matrimid-PCN-250 mixed matrix membranes displaying light-responsive gas separation and beneficial ageing characteristics for CO2/N2 separation.Sci Rep . 2018;8(1):1-14. doi:10.1038/s41598-018-21263-7

18. Mitra T, Bhavsar RS, Adams DJ, Budd PM, Cooper AI. PIM-1 mixed matrix membranes for gas separations using cost-effective hypercrosslinked nanoparticle fillers. Chem Commun . 2016;52(32):5581-5584. doi:10.1039/c6cc00261g

19. Cheng J, Wang Y, Hu L, Liu N, Xu J, Zhou J. Using lantern Zn/Co-ZIF nanoparticles to provide channels for CO2 permeation through PEO-based MMMs. J Memb Sci . November 2019:117644. doi:10.1016/j.memsci.2019.117644

20. Zhang H, Wang Y. Poly(vinyl alcohol)/ZIF-8-NH ${ }_{2}$ mixed matrix membranes for ethanol dehydration via pervaporation.AIChE J . 2016;62(5):1728-1739. doi:10.1002/aic.15140

21. Zhao X, Zhang H, Xu S, Wang Y. ZIF-8 membrane synthesized via covalent-assisted seeding on polyimide substrate for pervaporation dehydration. AIChE J . 2019;65(8). doi:10.1002/aic.16620

22. Ge B-S, Wang T, Sun H-X, Gao W, Zhao H-R. Preparation of mixed matrix membranes based on polyimide and aminated graphene oxide for $\mathrm{CO}_{2}$ separation. Polym Adv Technol . 2018;29(4):1334-1343. doi:10.1002/pat.4245

23. Kumar R, Zhang C, Itta AK, Koros WJ. Highly permeable carbon molecular sieve membranes for efficient CO2/N2 separation at ambient and subambient temperatures. J Memb Sci . 2019;583:9-15. doi:10.1016/j.memsci.2019.04.033

24. Zornoza B, Tellez C, Coronas J, Esekhile O, Koros WJ. Mixed matrix membranes based on 6FDA polyimide with silica and zeolite microsphere dispersed phases. AIChE J . 2015;61(12):4481-4490. doi:10.1002/aic.15011

25. Shen J, Zhang M, Liu G, Guan K, Jin W. Size effects of graphene oxide on mixed matrix membranes for $\mathrm{CO}_{2}$ separation. AIChE J . 2016;62(8):2843-2852. doi:10.1002/aic.15260

26. Shen G, Zhao J, Guan K, Shen J, Jin W. Highly efficient recovery of propane by mixed-matrix membrane via embedding functionalized graphene oxide nanosheets into polydimethylsiloxane. AIChE $J$ . 2017;63(8):3501-3510. doi:10.1002/aic.15720 
27. Denny MS, Moreton JC, Benz L, Cohen SM. Metal-organic frameworks for membrane-based separations. Nat Rev Mater . 2016;1(12):1-17. doi:10.1038/natrevmats.2016.78

28. Koros WJ, Zhang C. Materials for next-generation molecularly selective synthetic membranes. Nat Mater . 2017;16(3):289-297. doi:10.1038/nmat4805

29. Baker RW, Wijmans JG, Kaschemekat JH. The design of membrane vapor-gas separation systems. $J$ Memb Sci . 1998;151(1):55-62. doi:10.1016/S0376-7388(98)00248-8

30. Choi SH, Kim JH, Lee SB. Sorption and permeation behaviors of a series of olefins and nitrogen through PDMS membranes. J Memb Sci . 2007;299(1-2):54-62. doi:10.1016/j.memsci.2007.04.022

31. Merkel TC, Bondar VI, Nagai K, Freeman BD, Pinnau I. Gas sorption, diffusion, and permeation in poly(dimethylsiloxane). J Polym Sci Part B Polym Phys . 2000;38(3):415-434. doi:10.1002/(SICI)10990488(20000201)38:3<415::AID-POLB8>3.0.CO;2-Z

32. Lin D, Ding Z, Liu L, Ma R. Experimental study of vapor permeation of C 5 C 7 alkane through PDMS membrane. Chem Eng Res Des . 2012;90(11):2023-2033. doi:10.1016/j.cherd.2012.03.007

33. Rebollar-Perez G, Carretier E, Lesage N, Moulin P. Vapour permeation of VOC emitted from petroleum activities: Application for low concentrations. J Ind Eng Chem . 2012;18(4):1339-1352. doi:10.1016/j.jiec.2012.01.039

34. Gadipelli S, Travis W, Zhou W, Guo Z. A thermally derived and optimized structure from ZIF-8 with giant enhancement in CO2 uptake.Energy Environ Sci . 2014;7(7):2232-2238. doi:10.1039/c4ee01009d

35. Mao H, Zhen HG, Ahmad A, et al. Highly selective and robust PDMS mixed matrix membranes by embedding two-dimensional ZIF-L for alcohol permselective pervaporation. J Memb Sci . 2019;582:307-321. doi:10.1016/j.memsci.2019.04.022

36. Casco ME, Cheng YQ, Daemen LL, et al. Gate-opening effect in ZIF-8: the first experimental proof using inelastic neutron scattering. Chem Commun . 2016;52(18):3639-3642. doi:10.1039/C5CC10222G

37. Yan C, Li H, Ye Y, et al. Coordinatively unsaturated nickel-nitrogen sites towards selective and high-rate CO2 electroreduction. Energy Environ Sci . 2018;11(5):1204-1210. doi:10.1039/c8ee00133b

38. Yao J, Wang H. Zeolitic imidazolate framework composite membranes and thin films: Synthesis and applications. Chem Soc Rev . 2014;43(13):4470-4493. doi:10.1039/c3cs60480b

39. Zhu J, Li H, Hou J, Liu J, Zhang Y, Van der Bruggen B. Heteroepitaxial growth of vertically orientated zeolitic imidazolate framework-L (Co/Zn-ZIF-L) molecular sieve membranes. AIChE J . 2020;66(5). doi:10.1002/aic.16935

40. Masoomi MY, Morsali A, Dhakshinamoorthy A, Garcia H. Mixed-Metal MOFs: Unique Opportunities in Metal-Organic Framework (MOF) Functionality and Design. Angew Chemie Int Ed . 2019;58(43):1518815205. doi:10.1002/anie.201902229

41. Abednatanzi S, Gohari Derakhshandeh P, Depauw H, et al. Mixed-metal metal-organic frameworks. Chem Soc Rev . 2019;48(9):2535-2565. doi:10.1039/c8cs00337h

42. Fang M, Wu C, Yang Z, Wang T, Xia Y, Li J. ZIF-8/PDMS mixed matrix membranes for propane/nitrogen mixture separation: Experimental result and permeation model validation. J Memb Sci . 2015;474:103-113. doi:10.1016/j.memsci.2014.09.040

43. Song Q, Nataraj SK, Roussenova M V., et al. Zeolitic imidazolate framework (ZIF-8) based polymer nanocomposite membranes for gas separation. Energy Environ Sci . 2012;5(8):8359-8369. doi:10.1039/c2ee21996d 
44. Li R, Ren X, Feng X, Li X, Hu C, Wang B. A highly stable metal- and nitrogen-doped nanocomposite derived from Zn/Ni-ZIF-8 capable of CO2 capture and separation. Chem Commun . 2014;50(52):6894-6897. doi: $10.1039 / \mathrm{c} 4 \mathrm{cc} 01087 \mathrm{f}$

45. Mao H, Zhen HG, Ahmad A, Zhang AS, Zhao ZP. In situ fabrication of MOF nanoparticles in PDMS membrane via interfacial synthesis for enhanced ethanol permselective pervaporation. $J$ Memb Sci . 2019;573:344-358. doi:10.1016/j.memsci.2018.12.017

46. Xiang L, Sheng L, Wang C, Zhang L, Pan Y, Li Y. Amino-Functionalized ZIF-7 Nanocrystals: Improved Intrinsic Separation Ability and Interfacial Compatibility in Mixed-Matrix Membranes for CO2/CH4 Separation. Adv Mater . 2017;29(32):1606999. doi:10.1002/adma.201606999

47. Yang H, Nguyen QT, Ding Y, Long Y, Ping Z. Investigation of poly(dimethyl siloxane) (PDMS)-solvent interactions by DSC. J Memb Sci . 2000;164(1-2):37-43. doi:10.1016/S0376-7388(99)00187-8

48. Zhang C, Lively RP, Zhang K, Johnson JR, Karvan O, Koros WJ. Unexpected molecular sieving properties of zeolitic imidazolate framework-8. J Phys Chem Lett . 2012;3(16):2130-2134. doi:10.1021/jz300855a

49. Haldoupis E, Watanabe T, Nair S, Sholl DS. Quantifying Large Effects of Framework Flexibility on Diffusion in MOFs: $\mathrm{CH}_{4}$ and $\mathrm{CO}_{2}$ in ZIF-8. ChemPhysChem . 2012;13(15):3449-3452. doi:10.1002/cphc.201200529

50. Dewar MJS. A Molecular Orbital Theory of Organic Chemistry. I. General Principles. J Am Chem Soc . 1952;74(13):3341-3345. doi:10.1021/ja01133a038

51. De Angelis MG, Sarti GC. Solubility and diffusivity of gases in mixed matrix membranes containing hydrophobic fumed silica: Correlations and predictions based on the NELF model. Ind Eng Chem Res . 2008;47(15):5214-5226. doi:10.1021/ie0714910

52. Merkel TC, Bondar V, Nagai K, Freeman BD. Sorption and transport of hydrocarbon and perfluorocarbon gases in poly(1-trimethylsilyl-1-propyne). J Polym Sci Part B Polym Phys . 2000;38(2):273-296. doi:10.1002/(SICI)1099-0488(20000115)38:2<273::AID-POLB1>3.0.CO;2-X

53. Semenova SI. Polymer membranes for hydrocarbon separation and removal. J Memb Sci . 2004;231(12):189-207. doi:10.1016/j.memsci.2003.11.022

54. Okamoto K, Noborio K, Hao J, Tanaka K, Kita H. Permeation and separation properties of polyimide membranes to 1,3-butadiene and n-butane. J Memb Sci . 1997;134(2):171-179. doi:10.1016/S03767388(97)00128-2

55. Lin H, Freeman BD. Gas and vapor solubility in cross-linked polyethylene glycol diacrylate. Macromolecules . 2005;38(20):8394-8407. doi:10.1021/ma051218e

56. W. M. Haynes, David R. Lide TJB. CRC Handbook of Chemistry and Physics . 95th ed. Boca Raton, FL: CRC Press Taylor \& Francis Group; 2014.

57. Freeman BD. Basis of permeability/selectivity tradeoff relations in polymeric gas separation membranes. Macromolecules . 1999;32(2):375-380. doi:10.1021/ma9814548

58. Barrer RM. Permeability in relation to viscosity and structure of rubber. Trans Faraday Soc . 1942;38(0):322-330. doi:10.1039/tf9423800322 

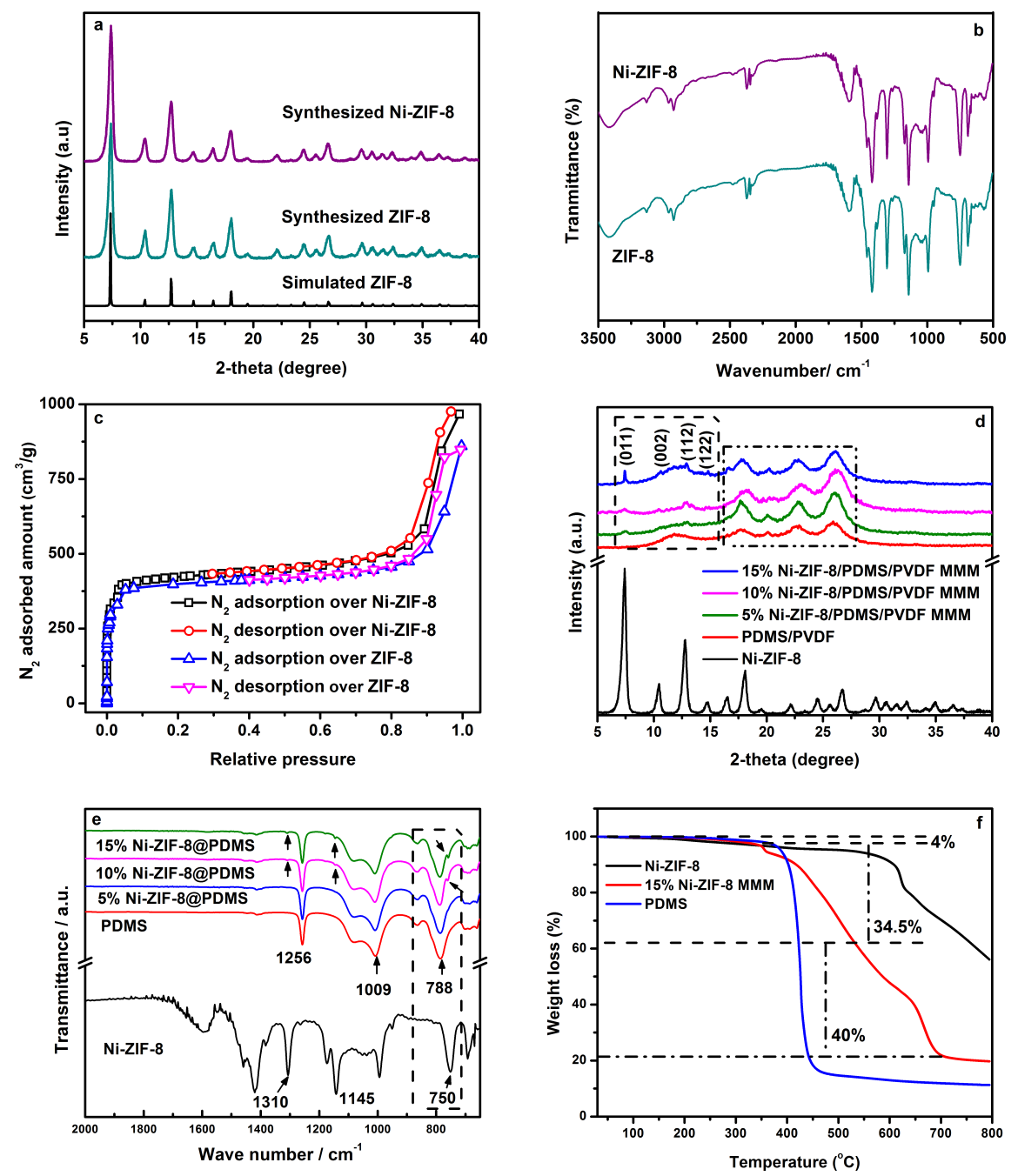

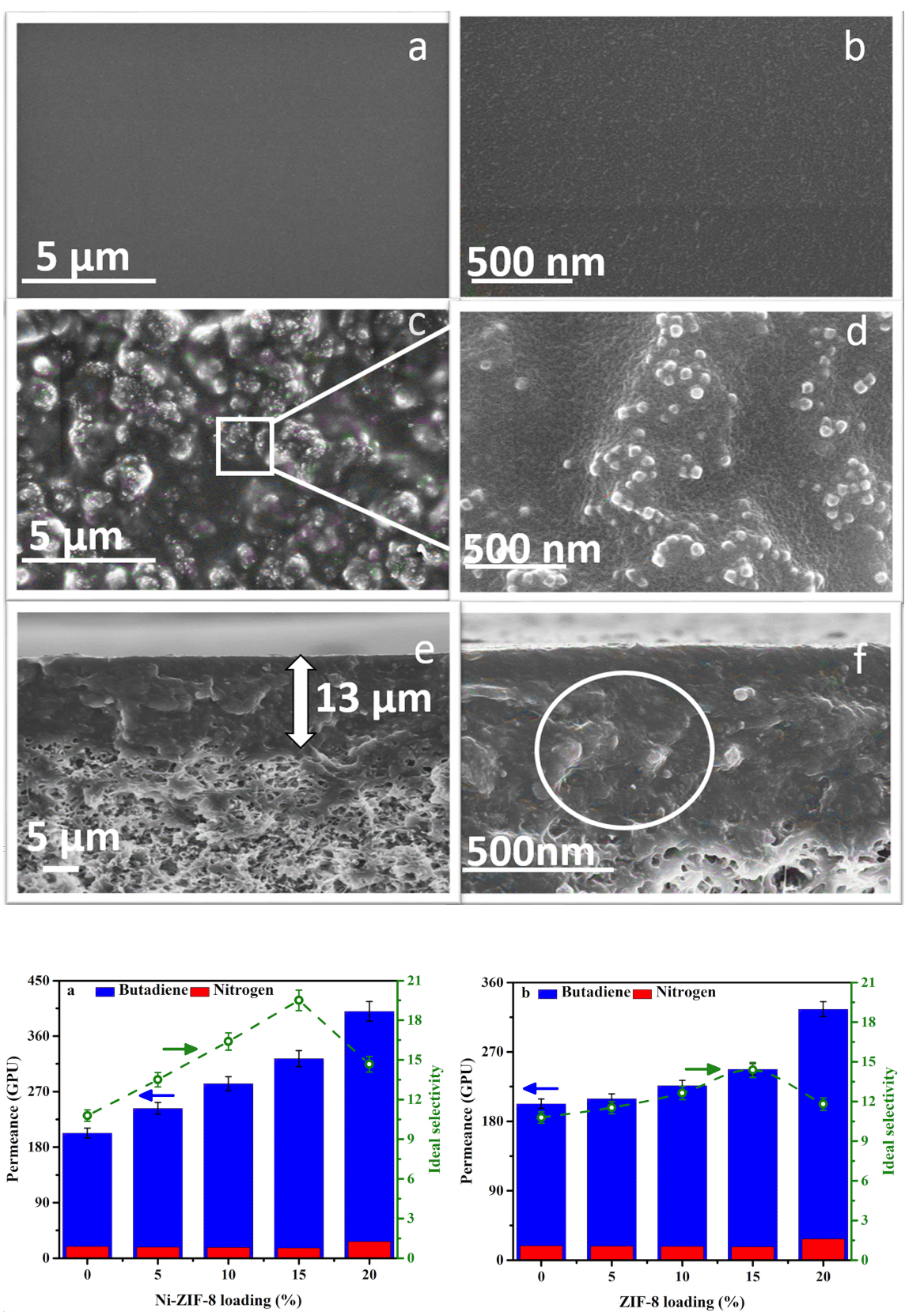

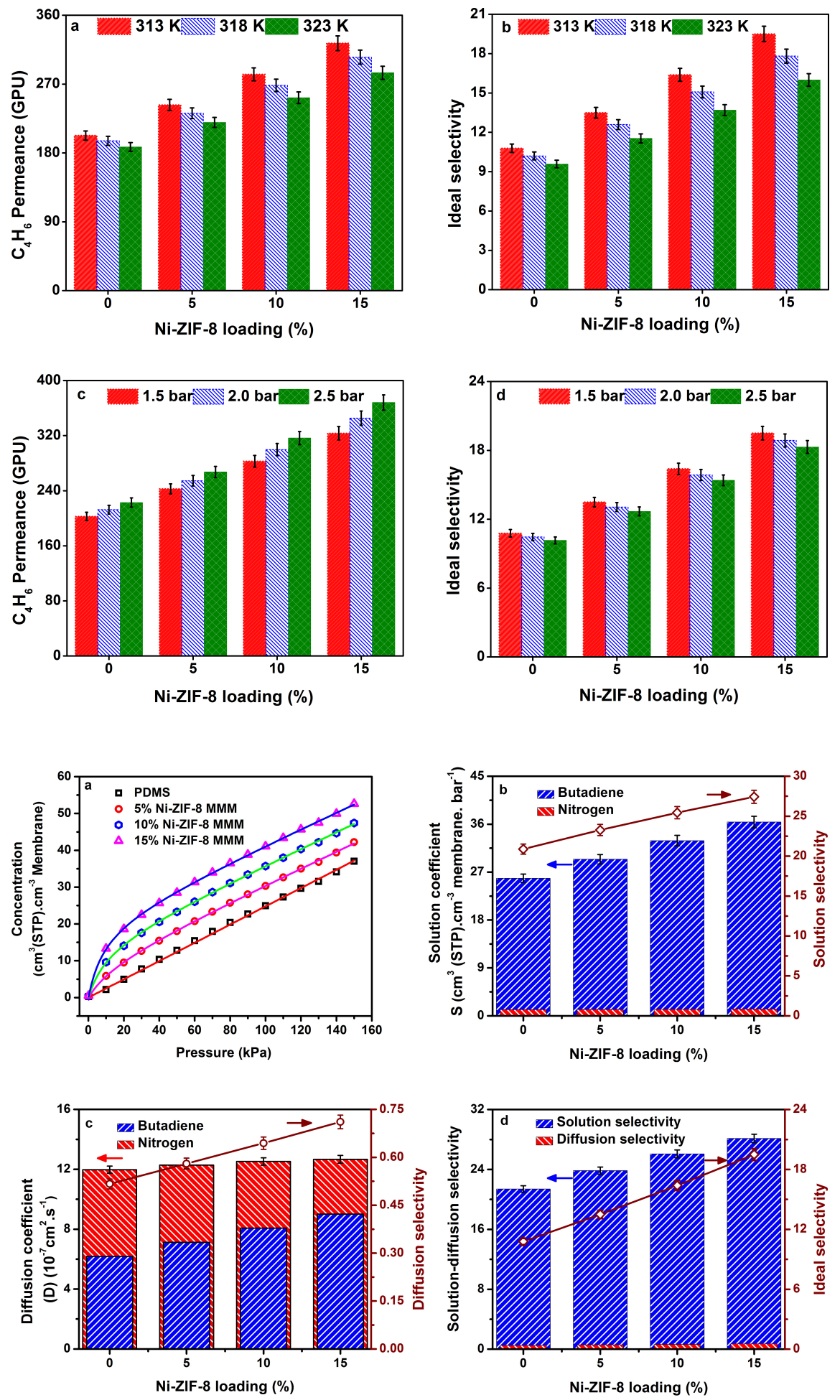

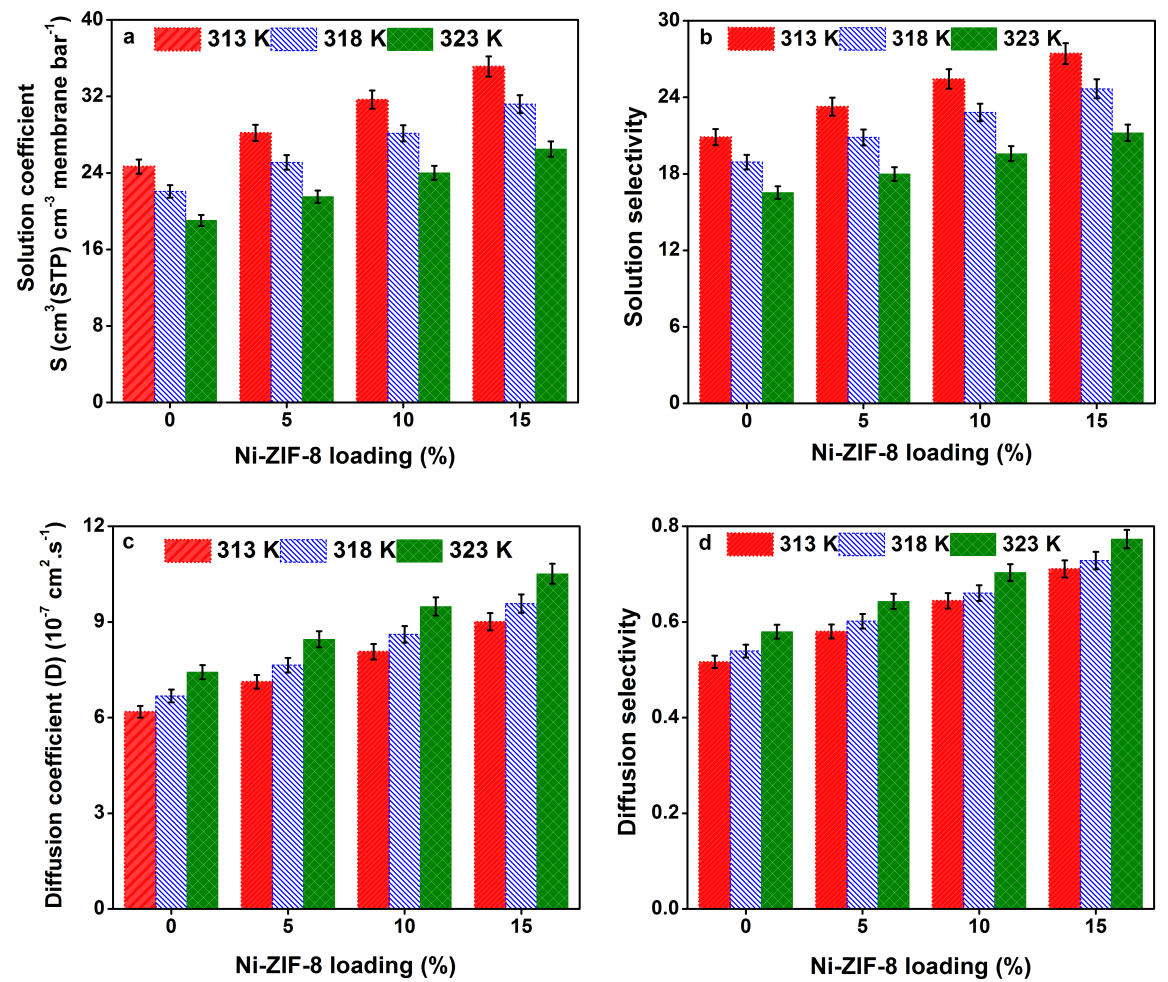

\section{Hosted file}

Table.docx available at https://authorea.com/users/325061/articles/453101-solution-diffusionproperties-of-c4h6-and-n2-molecules-in-zeolitic-imidazolate-framework-based-mixedmatrix-membranes 